\title{
Revisionen
}

Grundbegriffe der Literaturtheorie

\author{
Herausgegeben \\ von \\ Fotis Jannidis \\ Gerhard Lauer \\ Matías Martínez \\ Simone Winko
}

1

Walter de Gruyter · Berlin · New York

2003 


\title{
Regeln der Bedeutung
}

Zur Theorie der Bedeutung literarischer Texte

\author{
Herausgegeben \\ von \\ Fotis Jannidis \\ Gerhard Lauer \\ Matías Martínez \\ Simone Winko
}

Walter de Gruyter · Berlin · New York

2003 


\section{PETER STROHSCHNEIDER}

\section{Unlesbarkeit von Schrift}

\section{Literaturhistorische Anmerkungen zu Schriftpraxen in der religiösen Literatur des 12. und 13. Jahrhunderts}

Unter dem empörten Protest der internationalen (und das hieß in diesem Fall keineswegs bloß: im Wesentlichen der rwestlichen () Öffentlichkeit zerstörten im Frühjahr 2001 die Milizen der Taliban die beiden monumentalen Buddha-Statuen von Bamiyan in Afghanistan. ${ }^{1}$ Dabei handelte es sich um einen Vorgang, in welchem sich, habe ich recht verstanden, zwei kulturelle Alteritätskonstellationen verschränkten. Einerseits und gewissermaßen in einem Offensichtlichkeits-zusammenhang, auf den hin sie von den Taliban geradezu angelegt wurde, war die ikonoklastische Aktion Element eines religiösen Konfliktes, ${ }^{2}$ in welchem sich eine aktuelle, so genannte fundamentalistische Version des Islam und in Gestalt tausendfünfhundertjähriger steinerner Überlieferung der Buddhismus gegenüberstehen. Andererseits aber und gewissermaßen quer hierzu rücken die Ob-

1 Vgl. Neef: Besudelung; Hohmeyer: „Bist du Gott, so wehr dich«; Mazzoni: Augen. Für Anstiftungen und Diskussionsmöglichkciten während der Arbeit an diesem Artikel der vor dem 11. September 2001 im Manuskript fertig gestellt war - bin ich Beate Kellner, Ludger Lieb und Stephan Müller herzlich dankbar. In knapperer Form erscheinen meine Überlegungen auch in einem von Sybille Krämer herausgegebenen Band über >Perforativität und Medialitäts.

2 Genauso gut könnte freilich auch von einem politischen Konflikt gesprochen werden: weil in ihm nämlich die Begriffe des Politischen und des Religiösen nicht (oder jedenfalls nicht in einer der uns geläufigen Formen) gegeneinander ausdifferenziert sind. Zur aktuellen Theoriedebatte wäre etwa zu vergleichen Ottmann: Theologie und Assmann: Herrschaft. 
jekte wie die Subjekte des Zerstörungsaktes gemeinsam in eine Position der Alterität gegenüber jenen Formen der Rede und der Wirklichkeitskonstruktion, die den Einspruch der Weltöffentlichkeit getragen haben. Dieset Einspruch muss als barbarischen Vandalismus verurteilen, ${ }^{3}$ was sich selbst als Moment eines heiligen Krieges begreift und was aus analytischer Distanz zu beschreiben wäre als - momentan jedenfalls erfolgreicher Versuch des Nachweises der Überlegenheit des einen, eigenen Gottes gegenüber den fremden, alten Göttern. Paradoxerweise nämlich, so wird man sagen müssen, nahmen allein die Zerstörer den Status der Statuen als Formen der kultischen Gegenwärtigsetzung eines Göttlichen ernst. Die kritische Weltöffentlichkeit hingegen verstand die Buddha-Statuen als Bezeichnungen, die zum Bezeichneten substanzielle Beziehungen längst nicht mehr unterhalten: Zeugnisse ästhetisch-kultureller Überlieferung, Kunst- und Kultur-Denkmale. Nur als solche aber hätten die Statuen zum Gegenstand von - in einem systematischen Sinne musealisierender - Aufbewahrung werden können: Die Musealisierung von Kultbildern, von Kultgegenständen überhaupt ist nur um den Preis ihrer Zerstörung als Kultobjekte möglich. Als solche nämlich entziehen sich Kultgegenstände den Aufbewahrungs- und Gebrauchsformen für Kunst. ${ }^{4}$ Dass der zu ihrer Rettung vorgeschlagene Kauf der Buddhas von Bamiyan durch das New Yorker >Metropolitan Museum of Arts von den Taliban abgelehnt wurde, ist insofern systematisch schlüssig.

Es ist weniger der Zeichencharakter der Statuen als vielmehr eine Art von fortwährender Präsenz des falschen Gottes und seines tremendum ${ }^{5} \mathrm{im}$ dauerhaften Stein, die jenen Schrecken auslöst, der sich in zerstörerischer Gewalt entlädt. Umgekehrt setzt die Auffassung der Statuen als unter allen Umständen aufbewahrenswertes Kultur-Denkmal voraus, dass irgend ssubstanzielle Gehalter jenseits ihrer reinen Materialität in ihnen keineswegs anwesend sind. Als solche Kultur-Denkmale riefen die Statuen keinerlei Schrecken mehr hervor. Sie wären allenfalls Objekte eines ästhetischen oder kulturhistorischen (um nicht zu sagen: kulturtouristischen) Interesses, wären allein noch Bedeutungsträger - lesbar als Verweise auf Abwesendes, das sie selbst nicht sind: absente Götter, vergangene religiöse

3 Und es scheint mir die Vermutung nicht abwegig, dass das Assonanzengeflecht Taliban - Caliban - Kanibale hierzu undurchschaute Stützscmantiken beisteuert: die >Islamisten - der grobschlächtige Wilde aus Shakespeares ,Tempest، (vgl. hierzu auch Löwenthal: Calibans Erbe) - der Menschenfresser.

4 Vgl. Wyss: Panorama: »Das Kulturgut ist das Totem der aufgeklärten Gesellschaft, das Muscum dessen Tempel. [...] Solange die Magie der Macht in den Fetischen zu stecken scheint, herrscht der Bildersturma (S. 561). „Das Museum verwandelt das materielle Erbe der Vergangenheit von Fetischen in Kunstwerke" (S. 562).

5 Vgl. Otto: Heilige, bes. S. 13 ff.; vgl. dazu neuerdings Luhmann: Religion, S. $10 \mathrm{ff}$. 
Traditionen, längst verstorbene Künstler, fremde Ästhetiken, in Vergessenheit geratene Kulturtechniken usw.

Es gibt, so kann man sich nicht nur an dieser Episode klarmachen, Formen des Belangvollen und in diesem Sinne des Bedeutsamen, ${ }^{6}$ die nicht aus (zeichenhaften, semiotischen) Bedeutungen hervorgehen und die daher nicht in Akten der Auslegung allein behandelbar sind, die vielmehr auch physische Praxis erfordern; ${ }^{7}$ Gewalttätigkeit ist eine ihrer Möglichkeiten. Demgegenüber legt es die deutsche Sprache nahe, das Bedeutsame und das Bedeutungsvolle eng zu verknüpfen. Bedeutsam in dem Sinne, dass ihm Wichtigkeit und Wert eigne, ist - so scheint es - zunächst das Bedeutungsvolle, das Hermeneutische; daher für zwei unterschiedliche Sachverhalte (jedenfalls alltagssprachlich) ein Wort ausreichend scheint: Bedeutung. Auf seiner einen Seite freilich ist dieses Wort sozusagen ein Pluraletantum. Semantische Bedeutungen kommen im Plural vor und sie können strittig sein. Unter den epistemischen Bedingungen derzeitiger Literatur- und Kulturwissenschaft lässt sich solche Strittigkeit auf den mittlerweile scheinbar selbstverständlich gewordenen Sachverhalt als eine ihrer Möglichkeitsbedingungen zurückführen, dass die Träger solcher Bedeutungen, dass die Wörter und die Dinge der Welt Bedeutung nicht san sich - wie man sagt - shaben oder besitzenc. ${ }^{8}$ Sie sind gerade nicht mehr als sbedeutungsvolk zu denken. Bedeutung wird ihnen vielmehr zugeordnet.?

Bedeutungen sind Resultat von Bedeutungszuweisungen, von Lektüreprozessen, die wir auf der Grundlage von Unterscheidungen Unterscheidungen vornehmen. Sie gehorchen stets den elementaren semiotischen Gesetzen der Abhebung des Bedeutenden (Signifikant) vom Bedeu-

6 sBedeutsamkeit wird im Folgenden als ein ganz vorläufiger Verständigungsausdruck für die Relevanz, die funktionale Wichtigkeit, den 'Wert für ... s eines Sachverhalts, für das an ihm Belangvolle verwendet. Ich orientiere mich damit an einem Wortgebrauch, wie er etwa Erich Rothackers S Satz der Bedeutsamkeit، (Rothacker: Probleme, S. 117 ff.) zugrunde liegt und wie er sich von einem spezifisch hermeneutischen Konzept von 'Bedeutsamkeitr unterscheidet; zu letzterem vgl. etwa Heidegger: Sein, S. 18; Blumenberg: Arbeit, S. $68 \mathrm{ff}$.

7 Während das Museum physische Praxis gerade auszuschließen unternimmt: Die »Magie der Ermächtigung durch Berühren wird hinter Glas, in Vitrinen, durch Alarmanlagen gebannt木. Die Wahmehmung der Gegenstände verlagert sich durch Musealisierung wvom sHaptischen zum Optischen«. (Wyss: Panorama, S. 563, 562) Ein 'Zugriff ist dieserart nicht mehr möglich.

8 Substanzialistische Bedeutungskonzepte verfügen dementsprechend in diesem Rahmen gegenwärtig nur über relativ geringe Chancen auf Durchsetzung ihrer Plausibilitätsansprüche; vgl etwa Steiner: Gegenwart, und dazu nur Jauß: Erfahrung. Vgl. weiterhin Gipper: Art. Bedeutung; Lyons: Bedeutungstheorien; Meggle / Siegwart: Streit; Thürnau: Art. Bedeutung.

9 Vgl. Heidegger: Sein, S. 31 ff.; Wittgenstein: Untersuchungen, S. 41 ff. 
teten (Signifikat) sowie der sinverse[n] Relation von Anwesenheit und Abwesenheit ${ }^{10}$ : Etwas sbedeutet etwas anderes, was es selbst nicht ist. Weil Bedeutung von etwas stets Bedeutung für jemanden ist, implizieren Bedeutungszuweisungen zugleich die Unterscheidung von jemand und jemand anderem. Schließlich weisen sie immer diese, nicht jene Bedeutung zu. Und dabei wird dann weiterhin unterschieden zum Beispiel zwischen je nachdem - wahren und unwahren, funktionalen und dysfunktionalen, gültigen und ungültigen, adäquaten und inadäquaten, relevanten und irrelevanten Bedeutungen. Behandlungen von Sachverhalten unter Aspekten ihrer Lesbarkeit, Lektüren also differenzieren - in mehrfacher Hinsicht. Hierin sind sie, insofern es sich bei Bedeutungszuweisungen um Akte handelt - und damit ist keineswegs unterstellt, diese Akte müssten intentional oder explizit sein -, an Situationen (Kontexte) gebunden. Sie geschehen im Rahmen und in Abhängigkeit von Handlungs- und Kommunikationsstrukturierungen, die als Praxisbereiche individuelles und kollektives Handeln verknüpfen sowie die konkreten Kommunikationen und die allgemeinen Regeln und Ordnungen einer Kultur aufeinander beziehen. ${ }^{11}$ Regeln der Bedeutung können im Blick auf solcherart pragmatische Handlungsgefüge als Regeln der situationalen Bedeutungszuweisung aufgefasst werden. ${ }^{12}$

Demgegenüber sollte das Eingangsbeispiel von der Zerstörung der Buddha-Statuen durch die Taliban-Milizen darauf aufmerksam machen, dass bestimmte Sachverhalte unter bestimmten Umständen nicht allein im Hinblick auf ihre Bedeutungen und mithin ihre Lesbarkeit belangvoll werden, also: Bedeutsamkeit gewinnen können, sondern zugleich auch auf eine sozusagen bedeutungsferne Weise. Letzteres geschieht dann und insofern, als derartige Sachverhalte zum Gegenstand von Praxen werden, welche von (differenziellen) Bedeutungen auch absehen, welche weniger hermeneutisch verfahren als sinnhafte Prozesse lektüreförmiger Bedeutungszuweisung, sondern welche vielmehr sinnliche Akte sind: sensorische Wahrnehmungen und zumal, im Bereich der Nahsinne, physische Kontakte - in der körperlichen Berührung, in der Einverleibung, in der Zerstörung. In diesem Sinne könnte man sagen, es gebe Bedeutsames nicht

10 Assmann: Sprache, S. 238.

11 Vgl. Strohschneider: Situationen. - In ganz anderen Zusammenhängen wird die Situationalität von Bedeutung in der linguistischen Semantik diskutiert, vgl. etwa Cresswell: Weltsituation; Barwise: Situationen.

12 Das ermöglicht dann Anschlussfragen etwa danach, wie Bedeutungszuweisungen in räumlicher, zeitlicher und sozialer Hinsicht so aufeinander abgestimmt und wie sie relativ situationsabstrakt gemacht, wie sie, könnte man sagen, derart institutionalisiert werden, dass Formen kultureller Kohärenz und Persistenz sich herauszubilden vermögen. Fragen in solcher Richtung stelle ich hier hintan. 
ausschließlich nur im Hinblick auf, sondern möglicherweise auch unter Absehung von Bedeutungen ${ }^{13}$ - dasjenige nämlich, was berührt, gegessen, zerstört werden muss.

Der Hinweis auf solche Vorkommnisse in der Welt - oder vorsichtiger noch: auf derartige unhermeneutischer Dimensionen von Vorkommnissen und Praxen - impliziert nicht die These, es werde in oder mit ihnen Bedeutung gewissermaßen ausgelöscht oder es handele sich um einander ausschließende Alternativen - etwa im Sinne eines Gegensatzes: entweder lesende Zuweisung von Bedeutung oder sensorischer Akt. Gesagt sein soll lediglich dies: Dass ich der Welt nicht nicht Bedeutung zuweisen kann, das heißt noch nicht, dass jede Praxis in jeder Hinsicht Bedeutungszuweisung sei. Nicht alles, auch nicht alles Bedeutsame ist jederzeit, überall, unter allen Umständen, für jedermann und in jedweder Hinsicht lesbar. Wenn man dies aber sagen kann, dann folgt daraus für die angesprochenen Regeln, dass sie nicht allein die oben summarisch skizzierten Unterscheidungen regulieren müssen. Die Regeln stets situationaler Bedeutungszuweisung müssen auch (und systematisch sozusagen zuvor noch) die pragmatischen Situationen, die Praxen, die Hinsichten, in welchen ein Sachverhalt zum Gegenstand von Lektüren wird, unterscheiden von denjenigen, in welchen er unter (momentaner, lokaler, sektoraler) Absehung von seiner Lesbarkeit als Element einer sinnlichen Praxis bedeutsam ist. Regeln der Bedeutung regeln in diesem Sinne auch die Grenzen von Bedeutungszu-

13 Besonders stimulierend für eine Diskussion des systematischen Zusammenhangs der hier skizzierten Überlegungen wäre zumal Hans Ulrich Gumbrechts Konzept des Nicht-Hermeneutischen; vgl. insbesondere Gumbrecht: Nicht-Hermeneutische; Gumbrecht: Form; Gumbrecht: Reflections. - Dass Gumbrechts Entwürfe sich in einem Feld bewegen, in welchem es anscheinend wachsenden Bedarf gibt für Konzeptualisierungsmöglichkeiten von Momenten der Präsenz, der Gegenständlichkeit und Gegenwärtigkeit, der Verkörperung und der sinnlichen Wahrnehmung, dies indiziert eine gewisse Breite des (freilich auch hinsichtlich seiner Ausarbeitungsgrade wie seiner Plausibilitäten sehr heterogenen) terminologischen Angebotes, darunter etwa George Steiners »real presences«, Hans Beltings Unterscheidung von Kult-Bild und Kunst-Bild (sie gewann unter anderem erhebliche Bedeutung für Niklas Luhmanns Darstellung der »Ausdifferenzierung des Kunstsystems Didi-Hubermans Begriff des ,Visuellen, Stephen Jaegers Ausdrücke vom charismatischen Körper und vom charismatischen Text, Peter Czerwinskis Konzept der Gegenwärtigkeit oder institutionentheoretische Unterscheidungen von Anwesenheitszeichen mit ontologischem Status und Abwesenheitszeichen mit Vertragsstruktur. Vgl. hierzu Steiner: Gegenwart; Belting: Bild; Jauß: Erfahrung; Belting: Bild-Anthropologie; DidiHuberman: Bild, S. 19 ff.; Jaeger: Envy; Jaeger: Charismatic Body; Czerwinski: Abstraktion; Czerwinski, Gegenwärtigkeit; Rehberg: Weltrepräsentanz; Quast: Hand-Werk. - Nicht übersehen sollte man aber freilich, dass solche Konzepte zugleich eine interpretationstheoretische Vorgeschichte haben, die mindestens zurückreicht bis zu Sontag: Against Interpretation; vgl. hierzu auch Spree: Kritik. 
weisungen, die Grenzen der Lektüre. Welchem Sachverhalt in welcher Hinsicht überhaupt Bedeutung attribuiert wird, dies unterliegt nicht weniger kulturellen Regulierungen als die Frage, wer welche Bedeutungen wann, wo, unter welchen Bedingungen und für wen attribuiert. Solche Regulierungen können also insofern auch Grenzen der Bedeutung konstituieren - oder wiederum vorsichtiger: Grenzen der Relevanz von Bedeutungen -, Grenzen der Lesbarkeit also, jenseits welcher es auf die Unterscheidungsprozesse der Bedeutungszuweisungen (- momentan, an dieser Stelle, in der gegebenen Situation, hinsichtlich spezifischer Sachverhaltsmerkmale usw. $\rightarrow$ nicht ankommt.

Um solche Momente von Unlesbarkeit ist es den nachfolgenden Bemerkungen zu tun. Dies freilich nicht in einer grundsätzlichen und theoretisch explizierenden Weise, sondern im Ausgang von konkreten Textbeispielen aus dem Bereich der mittelalterlichen volkssprachigen Literatur. Es mag daher in mehrerlei Hinsicht nicht unangemessen sein, wenn ich zur Erläuterung der Möglichkeit einer nicht an die Bedeutungszuweisungen von Lektüreprozessen gebundenen Bedeutsamkeit zunächst an jene Manifestation erinnere, die in der europäischen kulturellen Tradition unzweifelhaft zentral ist: die eucharistische Transsubstantiation. Im eucharistischen Vollzug, im liturgischen wie systematischen Zentrum der deswegen heilig zu nennenden Messe wird gemäß katholischer Dogmatik seit dem Hochmittelalter in der Wandlung der Leib Christi für die Teilnehmer reak und total im Brot, sein Blut rreak und total im Wein spräsent..14 Die Gaben von Brot und Wein werden durch die Konsekration substanziell15 etwas, was sie vorher nicht waren. Im Moment der eucharistischen Kommunion sind die Hostie und der Messwein als nicht semiotische, sondern somatische Sachverhalte daher in dem angedeuteten Sinne unlesbar: Insofern sie Gegenstand von Einverleibungen sind, werden sie hier gerade nicht Gegenstand von Kommunikation, von hermeneutischer Praxis, also Träger von Bedeutung. Diejenigen, die an der heiligen Handlung im Modus der Un-

14 Vgl. Denzinger: Enchiridion Symbolorum, Nr. 355, 430 usw., sowic cbd. Nr. 465, 698, $715,877,884,997$, zum T'erminus iI ransubstantiation; Neunheuser: Eucharistie, S. 24 ff.; Jorissen: Entfaltung; Betz: Art. »Eucharistie«, hier S. 380 f., 383, 388 f.; Feld: Verständnis; Iserloh: Art. Abendmahl, bes. S. 91 ff; Hörisch: Brot; Gumbrecht: Form, S. $40 \mathrm{ff}$.; Laarmann: Transsubstantiation.

15 Seit Guitmund von Aversa (De corporis et sanguinis Domini veritate [PL 149, Sp. 1427-1512]: cntstanden zwischen 1073 und 1078) lautet der theologische terminus techntcus substantialiters. 
mittelbarkeit ${ }^{16}$ partizipieren, die Teilnehmer sozusagen im sInnern des kultischen Vollzugs sind in der angedeuteten Weise außerhalb der Ordnungen von Bedeutungszuweisungen, insoweit sie eucharistisch skommunizieren//konsumieren. Es ist dies ein - je nach gewähltem Diskurs - sakramental-gnadenhaftes, wundersames, magisches Geschehen von gar nicht zu überschätzendem Gewicht - es hätte sonst nicht über die Jahrhunderte der so genannten Glaubensspaltung hinweg (und darüber hinaus) zur Leitdifferenz der gesellschaftlichen Semantiken der europäischen Kulturen dienen können.

Dass für Beobachter der eucharistischen Kommunion die Hostie und der Wein davor, zugleich oder danach demgegenüber auch Objekt von Kommunikationen, von Bedeutungszuweisungen und hermeneutischer Anstrengung sein können (und dass man dann etwa von Theophagie sprechen kann), das bringt zwar für solche Beobachter, darunter zum Beispiel Theologen oder Mediävisten, spezifische theoretische und methodische Aufgabenstellungen mit sich. Es bestimmt indes nicht den Status der konsekrierten Gaben im Akt der eucharistischen Einverleibung selbst. Und um schließlich auch Missverständnissen aus der Gegenrichtung vorzubeugen: Selbstverständlich ist der eucharistische Vollzug stets auch eine Erinnerungsfeier, in ihm werden Bedeutungen, Repräsentationen, Zeichenprozesse unterschiedlichster Art produziert, so dass man sagen könnte, nicht einmal die eucharistische `Realpräsenz« Gottes sei ১reine〈 Präsenz in dem Sinne, dass sie nicht immer auch mit einer Fülle hermeneutischer Akte verbunden sei. Doch würde die Rede über die somatische $>$ Realpräsenz Christi in der Eucharistie deren spezifischen Status verfehlen, wenn sie nicht analytisch auseinander nähme, was im unmittelbaren Vollzug ungeschieden ist: Kategorial anders als in aller hermeneutischen Praxis des Lesens ist die im Akt der eucharistischen Kommunion gegebene göttliche Realpräsenzı jedenfalls in gewisser Hinsicht formal dadurch als unlesbar bestimmt, dass sie nicht unter den semiotischen Gesetzen der Zeichendifferenz und der Präsenz-Absenz-Inversion steht: Dass der Leib Christi in der eucharistischen Oblate real gegenwärtig sei, dies besagt nicht, dass er anderweit abwesend wäre.

$\mathrm{Zu}$ den Regeln der Bedeutung wären also - wie ich meine, und jedenfalls im Kontext auch historischer Erkenntnisinteressen - zugleich jene Distinktionsregeln zu zählen, in denen etwa institutionell gesichert ist, dass Brot und Wein unter definierten liturgischen Spezialbedingungen nicht als Gegenstand von Lektüreakten, Entzifferungen und Bedeutungs-

$16 \gg I m$ Erlebnismodus der Unmittelbarkeit wird jede explizite Unterscheidung (zum Bcispiel die nach Zeichen und Bezeichnctem) und damit auch die Unterscheidung von Unmittelbarkcit und Mittelbarkeit zum Ausnahmefall« (Luhmann: Kunst, S. 18). 
zuweisungen, sondern als Objekte von Einverleibung Bedeutsamkeit gewinnen. Unterscheidungsregeln allerdings zeigen Unterscheidungsbedarf an, und dieser besteht nicht allein in dem besonders prominenten Fall der eucharistischen Hostie. $\mathrm{Zu}$ jenen Sachverhalten, die unter bestimmten Umständen und in bestimmten Hinsichten nicht als lesbare zu behandeln sind, die dann nicht Absentes zeichenhaft repräsentieren, sondern es jenseits der inversen Bezogenheit von Gegenwärtigkeit und Abwesenheit vielmehr präsent machen, konnten (und können) auch Dinge gehören, die heute im Sinne einer Generalunterstellung mit der allergrößten Selbstverständlichkeit nur für Träger von Bedeutungen und Lektüreobjekte gehalten werden mögen. $\mathrm{Zu}$ nennen wären hier etwa auch Bilder, weswegen auf lange hin selbst die Kult-rIkonen aus den Zeitaltern vor der Entstehung der Kunst ${ }^{17}$ so behandelt wurden, als hätte sich auch in ihnen bereits die uns selbstverständliche unüberbrückbare Kluft zwischen (anwesendem) Abbild und (abwesendem) (Ur-)Bild aufgetan; freilich konnten unter dieser Voraussetzung zum Beispiel das göttliche Bildverbot des Dekalogs (Exodus 20,4) ${ }^{18}$ oder Ikonoklasmen ${ }^{19}$ kaum zureichend verständlich werden. Ein anderer derartiger Sachverhalt, den man in der Regel allein in seiner Lesbarkeit auffasst - und derjenige, auf den sich meine kasuistischen Überlegungen am Beispiel zweier hochmittelalterlicher Texte im folgenden konzentrieren -, ist die Schrift. 20

17 Vgl. Belting: Bild; Jauß: Erfahrung; Czerwinski: per visibilia ad invisibilia; DidiHuberman: Bild; vgl. auch oben Anm. 13.

18 Vgl. Assmann: Religion, S. 96 ff.; vgl. auch Assmann: Herrschaft, S. 157 ff.

19 Vgl. Cancik: Art. Ikonoklasmus; Jaeger: Body, S. 118; Assmann: Religion, S. 96 ff.; reichhaltiges Material, jedoch ohne kategoriale Unterscheidung von Kult- und Kunstbild, bei Warnke ( $\mathrm{Hg}$ ): Bildersturm. Die eingangs erwähnte Zerstörung der BuddhaStatuen im afghanischen Bamiyan ist nur das einstweilen jüngste und sicher nicht das letzte Beispiel für eine fortdauernde Wirksamkeit der magischen Macht der Kultbilder (vgl. auch Gladigow: Art. Kultbild). Sie lässt überdies deutlich werden, wie wenig also die hier angeschnittenen Fragen ins Innere historiographischer Elfenbeintürme eingekapselt werden können.

20 》acques Derridas verallgemeinerter und radikalisierter metaphorischer Gebrauch des Ausdrucks s Schrift wist als wichtig anerkannt - als so wichtig, daß er eine eigene Terminologie verdient" (Luhmann: Schrift, S. 350). Der Ausdruck schriftı wird daher im Folgenden - wie der zugehörige Ausdruck Lektüre - in seiner allgemeinsprachlichen Bedeutung gebraucht. Freilich versteht sich, dass die strukturalistisch-poststrukturalistischen Konzepte der écriture und der lecture keineswegs folgenlos bleiben für das, was man an Schrift (im üblichen Sinn) beobachten kann, vgl. hierzu Gumbrecht / Pfeiffer (Hg.): Schrift; Koschorke: Körperströme, S. 323ff, Campe: Art. Lecture. Auf eine theoretische Generalisierung der im Folgenden angestellten literarhistorischen Einzelfallbemerkungen zu Funktionsmöglichkeiten von Schrift jenseits ihrer Lesbarkeit verzichte ich; vgl. aber etwa Wellberry: Äußerlichkeit. 
Schrift ist lesbar. Sie transportiert Bedeutungen und funktioniert also über Verweisung, über Referenz. Sie setzt überdies zugleich auch insofern auf Absenz, ${ }^{21}$ als sie dem Schreiber Kommunikation mit abwesenden Lesern ermöglicht und - hernach - beim Leser den Schreiber als einen Abwesenden repräsentiert, dessen Rede als eine längst verklungene sie gleichwohl verstehbar macht. Schrift löst das Wissen und die Rede vom Körper, sie ist ein Instrument, Kommunikationssituationen beinahe beliebig in Raum und Zeit auszudehnen, sie ermöglicht es damit, Zeit als abgelaufene in die Kommunikation einzubauen und nötigt dieserart auch dazu, Kommunikation unter Anwesenden (Interaktion ${ }^{22)}$ von Kommunikation unter Abwesenden zu unterscheiden. Bei all dem bedeutet Schrift immer etwas, was sie selbst nicht ist, sie verweist über das ¿Zeigfeld ${ }^{23}$ der Interaktion hinaus auf Abwesendes: Schrift hält, indem sie Abwesendes repräsentiert, die Abwesenheit des Abwesenden als solche präsent, die Abwesenheit dessen mithin, worum es gegenwärtig geht, und dessen, der sich gegenwärtig äußert. Kaum wäre daher ein (analytisch gehaltvoller) Schriftbegriff denkbar, der ohne ein Konzept von Bedeutung auskommen könnte. Wie nichts sonst außer der Sprache, welche sie graphisch zu speichern gestattet, scheint Schrift in die Sphäre von Bedeutungen zu gehören.

Man könnte wohl auch so sagen: Die Lesbarkeit der Welt ${ }^{24}$ ist zusammen mit Heilsgeschichte und Naturallegorese in der modernen Welt gewissermaßen aus dem Blick geraten, ${ }^{25}$ die Lesbarkeit des Körpers ${ }^{26}$ hat sich seit dem Mittelalter in vielen Hinsichten historisch gewandelt. ${ }^{27} \mathrm{Hin}-$ gegen: Wenn sich etwas von selbst zu verstehen scheint und heutzutage als prinzipielle, historischem Wandel seit der Erfindung der Schrift selbst nicht mehr unterworfene Tatsache behandelt werden kann, dann ist das die Lesbarkeit von Schrift. Dies wirkt so vollständig selbstverständlich, dass es sich in idiomatischen Ausdrücken verfestigt hat: Bedeutungen

21 Zu dieser Kategorie vgl. Ernst: Art. Absenz.

22 Vgl. Luhmann: Sozialsysteme; Kieserling: Kommunikation.

23 Vgl. Bühler: Sprachtheorie, S. $79 \mathrm{ff}$.

24 Vgl. Blumenberg: Lesbarkeit; Ohly: Schriften. Zum Begriff des Lesens zuletzt so knapp wie konzise Aust: Art. Lesen.

25 Nur um freilich gegenwärtig in Gestalt der Herstellung der Lesbarkeit von genetischen 'Codes, dessen also, was in bestimmten Diskursen für den Inbegriff von »Naturı gehalten werden darf, ebenso wiederzukehren wie in der seit dem linguistic turn neu etablierten Lesbarkeit aller Kultur; vgl. hierzu neuerdings Neumann / Weigel (Hg.): Lesbarkeit.

26 Seine Schönheit oder Hässlichkeit, seine Gestik und Mimik (vgl. zuletzt Egidi [u.a.] [Hg.]: Gestik), aber auch Tätowierungen (vgl. Oettermann: Zeichen; Hahn: Handschrift), Narben (vgl. Küsters: Buchstabe), Figurenalphabete (vgl. Harms: Buchstabenkörper), usw.

27 Vgl. Wenzel: Partizipation; Wenzel: Hören. 
werden szugeschrieben<; und selbst groß angelegte enzyklopädische Handbücher über Schrift und Schriftlichkeit thematisieren allenfalls am Rande die im Folgenden interessierenden Funktionsmöglichkeiten von Schrift über ihre Lesbarkeit hinaus. ${ }^{28}$ Doch ist dieser selbstverständliche Begriff von Schrift ein partikularer. Denn selbst im Bezug auf Schrift kann sich in bestimmten Situationen unter bestimmten Aspekten Entscheidungsbedarf dahingehend ergeben, ob sie eher als lesbares Objekt von Bedeutungszuweisung und Medium der Repräsentation von Abwesendem behandelt werden solle, oder ob vielmehr ein Praxiszusammenhang vorliege, in welchem Schrift als Instrument der Herstellung von unmittelbarer Präsenz funktioniere. Diese letztgenannte Option ist im Folgenden an Beispielen zu zeigen; solchen zwar, die nicht den Traditionen hieroglyphischer und kabbalistischer Schriftverwendung ${ }^{29}$ entstammen. Es geht also um Konstellationen einer spezifischen Unlesbarkeit von Schrift ${ }^{30}$ und um Prozesse ihrer Lesbarmachung (sowie umgekehrt der Unlesbarmachung von izunächste lesbarer Schrift). ${ }^{31} \mathrm{Da}$ es freilich eine hermeneutische Anstrengung ist, welcher es um die genannten Konstellationen und Prozesse geht, so wird sie gut tun, jene Selbstverständlichkeit zu suspendieren, mit welcher Schrift als Gegenstand von Lektüre, als Projektionsfläche von Bedeutungszuschreibungen aufgefasst wird.

Die folgenden Lektüren befragen zwei alte Texte - die Mariendichtung des Priesters Wernher und die Alexius-Legende Konrads von Würzburg-

28 Vgl. Günther / Ludwig (Hg): Schrift. - Worum es im Folgenden geht, ist hier über das Inhaltsverzeichnis kaum zu finden und auch im Sachregister fehlen entsprechende Lemmata. Der sachlich einschlägige Artikel von Manfred Geier versteckt das Thema unter dem Schlagwort von den "Sekundäre[n] Funktionen von Schriftu (ebd., S. 678686). Doch in welchem Sinne - logisch? historisch? gar frequenzstatistisch? - sollte man (um nun diesen Ausdruck durch die Seitentür einzuführen:) magische Funktionen von Schrift als ssekundär auffassen können? Vgl. Heidegger: Sein, S. 81 f.; Haarmann: Universalgeschichte, S. $69 \mathrm{ff}$.

29 Vgl. Assmann / Assmann: Art. Schrift, hier Sp. $1426 \mathrm{f}$.

30 In systematischer Hinsicht verglichen werden könnten jene monastischen Schriftpraxen im Mittelalter, im Bezug auf welche Ivan Illich (Illich: Weinberg) von Schrifttexten als "Partitur für fromme Murmler« (S. 8) gesprochen hat; vgl. auch Illich: Prägung. Vergleichbare Momente im Zusammenhang einer vorwiegend neuzeitlichen "Phänomenalität von Literatur skizziert zum Beispiel Schlaffer: Umgang mit, Literatur, hier S. $10 \mathrm{ff}$ - Geheimschriften übrigens (vgl. Costamagna: Kryptographie) gehören freilich nicht hierher: Sie sind möglicherweise unverstehbar, aber nicht unlesbar: Frst beim - scheiternden oder gelingenden - Versuch der Entzifferung kann man sie als Kryptographien identifizieren. Man muss sie also jedenfalls hermeneutisch behandeln.

31 Dabei wird sich nach dem bisher Gesagten verstehen, dass hier freilich nicht eine kategoriale Unlesbarkeit von Schrift gemeint sein kann, sondern lediglich eine von mehreren Funktionsdimensionen von Schrift, und dies an einem epistemologischen Ort, an welchem (gleich derjenigen der Welt, vgl. oben Anm. 24) die Lesbarkcit von Schrift immer schon metaphysisch garantiert ist. 
nach vergangenen kulturellen Wissensformen darüber, dass und wie die Lesbarkeit von Schrift produziert werde oder sistiert werden könne: wie also - könnte man sagen - Schrift in die Bedeutungsferne verschoben oder von dort gewissermaßen auch wieder zurückgeholt werde in die differenziellen Ordnungen der Zeichen und Repräsentationen. Dabei ist es schwerlich ein Zufall, dass die literarischen Beispiele, an denen entlang ich argumentiere, einer entscheidend durch Vokalität ${ }^{32}$ gekennzeichneten Kultur entstammen, einer Kultur mithin, in welcher die Schrift auch noch als Lektüregegenstand relativ körpernah funktioniert. ${ }^{33}$ Demgegenüber lässt sich zugleich sagen, dass die im Folgenden interessierenden skripturalen Funktionsmodalitäten in modernen Gesellschaften kaum je mehr hegemoniale Geltung etlangen. ${ }^{34}$ Am einfachsten lassen sie sich daher vielleicht als sschriftmagischer Funktionen klassifizieren - weil und obwohl ein solcher Ausdruck typischerweise als in sich widersprüchlich aufgefasst wird: >Magisch wäre gerade die Aufhebung jener inversen Relation von Absenz und Präsenz $z^{35}$ und jener Zeichendifferenz, die als ein Grundgesetz von Schrift begreifbar sind. In diesem noch sehr vorläufigen Sinne ist hier von Schriftmagie die Rede - und da es sich um eine literaturgeschichtliche Skizze handelt: von literarischer Rede über Schriftmagie. ${ }^{36}$ Es geht um

32 Vgl. Zumthor: Lettre; Schaefer: Vokalität.

33 Vgl. die jüngste Übersicht (mit weiterer Literatur) von Wenzel / Lechtermann: Repräsentation, bes. S. 197 ff. - Zwar haben die Diskussionen um Mündlichkeit und Schriftlichkeit in den letzten Jahren und Jahrzehnten nicht zuletzt auch in der Mediävistik eindrücklich gezeigt, dass Schrift für die mittelalterlichen laikalen Aristokratien zunächst subsidiär ist gegenüber direkter Interaktion von Angesicht zu Angesicht (vgl. Anm. 32). Doch bedeutet dies nicht zugleich auch, Schrift habe hier einen einheitlichen oder gar einfach zu beschreibenden Status. Insofern verstehen sich die folgenden Beobachrungen auch als ein kleiner Beitrag dazu, gegenüber einem öfters wenig differenzierten Schriftbegriff, wie ihn die mediävistischen Philologien (ähnlich dem Textbegriff) nicht selten ohne weitere Historisierung aus den epistemischen Bedingungen entwickelter moderner Schriftkulturen ableiten, Aspekte der Differenziertheit und damit auch der Alterität von Schrift und Schriftgebrauch in den volkssprachigen Kommunikationszusammenhängen laikaler Aristokratien herauszuarbeiten.

34 Die im Folgenden diskutierten Texte stammen demgegenüber, dies soll vorab festgehalten werden, keineswegs aus residualen oder marginalen Bereichen vergangener mittelalterlicher Gesellschaften, sondern aus ihren kulturellen Zentren. Es sind - und wiewohl es sich um religiöse Sujets handelt - freilich zugleich nicht Textbeispiele, die (sozusagen auf der anderen Seite) kultisch-liturgischen Sonderräumen eines magischen Schriftgebrauchs zugeordnet wären. Die Gedichte gehören vielmehr literarhistorisch ganz in die Zusammenhänge dessen, was man überhaupt eine volkssprachige Literatur im Ausdifferenzierungsprozess spezifisch laikaler Kommunikationsräume nennen könnte.

35 Vgl. zu diesem Wortgebrauch auch Gumbrecht: Reflections, S. 72.

36 Schriftmagie, nicht Buchstabenmagie: nicht also die magischen Funktionen einzelner Graphismen, vgl. dazu Geier: Funktionen; Czerwinski: Schrift. - Finiges Material zur 
literarische Texte, die punktuell, situational oder in bestimmten Hinsichten für sich eine Bedeutsamkeit beanspruchen, welche gerade im Abstand zu differenziellen Bedeutungszuweisungen sich konstituiert: eine Bedeutsamkeit, die aus der Differenz zum Differenzierten erwächst.

\section{3.}

Ich beginne mit dem jüngeren der beiden Beispieltexte. Für laikale Mitglieder der Basler Stadtaristokratie, so weist es eine Stifternotiz in seinem Text aus ${ }^{37}$, erzählt Konrad von Würzburg im dritten Drittel des 13. Jahrhunderts die Geschichte des heiligen Asketen Alexius, der der prächtigmächtigen Welt der römischen Ritter und Senatoren entflieht, um im syrischen Edessa als frommer Almosenempfänger seine 'Tage hinzubringen, der später indes in seine Heimatstadt zurückkehrt und so unerkannt wie verachtet als Bettler siebzehn Jahre unter einer Treppe seines Vaterhauses lebt, bevor er dort eines seligen Todes stirbt. Diese im Mittelalter vor allem in den linguae vernaculae außerordentlich weit verbreitete, in ihrem epischen Gerüst gleichwohl recht stabile Legende eines kultgeschichtlich wenig prominenten Heiligen ${ }^{38}$ wird von dem Baseler Erzähler, was hier nicht im einzelnen verfolgt werden $\mathrm{kann}^{39}$, vor allem dahingehend akzentuiert, dass die Heiligkeit ihres Protagonisten sich nicht allein in seiner ethischen Virtuosität und in den mit ihm verbundenen Mirakeln manifestiert; vom einen wie vom anderen wird hier nur sehr summarisch berichtet. Alexius erscheint vielmehr dominant als ein Heiliger, der stets verschwindet: der sich der Beobachtung und Repräsentation entzieht, den

Schriftmagie im Mittelalter bieten Enst: Sprachmagie, hier S. 150 ff.; Ernst: Formen der Schriftlichkeit, hier S. 347 ff., sowie Beiträge bei Ganz (Hg.): Repräsentationsobjekt; Classen (Hg): Book; zuletzt Schreiner: Buchstabensymbolik; Schreiner: Buch; Schreiner: Buchstaben; Griese: Gebrauchsformen, hier S. $194 \mathrm{ff}$ - Schneisen durch die längst unabsehbar gewordenen Diskussionsgeflechte über dem Begriff der Magie legen etwa: Kippenberg / Luchesi (Hg.): Magie (die Grundzüge der dortigen Einleitung sind wieder aufgenommen bei Kippenberg: Art. Magie); Macho: Bemerkungen; Zingerle: Der mmoralische Körper«; Luhmann: Gesellschaft, S. 644 ff.

37 Konrad von Würzburg: Alexius, hier 1392 ff. Vgl. dazu Jackson: Konrad, hier S. 210 ff.; Leipold: Auftraggeber, bes. S. 65 ff.; Bumke: Mäzene, S. 262, 288 f.; Feistner: 'Typologie, S. 157 ff. u.ö.

38 Vgl. Palmer: Prosabearbeitung, hier S. 159; sowie zur Kultgeschichte des Alexius Sckommodau: Alexius, bes. S. 165 ff., 187 f.; Mölk: 'Chanson de saint Alexisc, bes. S. 342 ff.; Jackson: Legends, S. 6 ff.; weiter die Hinweise bei Storey: Bibliography.

39 Vgl. aber Strohschneider: Textheiligung, wo versucht wird, die hier ausschnittsweise skizzierte Interpretation ausführlicher und im Zusammenhang zu begründen. Zur Interpretation von Konrads, Alexiuse ist weiterhin zu vergleichen Wyss: Theorie, S. 216 ff.; Jackson: Konrad; Jackson: L.egends; Feistner: Typologie, S. 185 ff. 
man immer (und stets vergeblich) suchen muss, dessen Gottverähnlichung erzählt wird zumal als Heraustreten aus der Immanenz der Welt. Die poetischen Verfahren von Konrads Legende stellen in besonders prägnanter Weise jenes allgemeine poetologische Grundlagenproblem legendarischen Erzählens im Mittelalter aus, welches darin besteht, dass dieses seinen reigentlichen Gegenstand im Akt der narrativen Repräsentation gerade als nichtrepräsentierbaren konstituiert: die Heiligkeit des Heiligen. ${ }^{40}$

In diesem hier nur stichwortartig erinnerten Zusammenhang operiert nun auch jenes epische Moment, um dessentwillen ich auf diese Erzählung zu sprechen komme. Die Alexius-Legende - auch in der Version Konrads von Würzburg41 - bearbeitet das Problem der Nichtrepräsentierbarkeit des Heiligen, indem sie diesen sich selbst repräsentieren lässt. Erzählt wird dies folgendermaßen: Als er spürt, dass er bald würde sterben müssen, erbittet der Asket unter der Treppe des römischen Senatorenpalastes von einem der Diener seines Vaters Schreibzeug und notiert damit alliu sîniu dinc (753). Er schreibt also sozusagen seine Autobiographie auf, ${ }^{42}$ und er tut dies mit einiger Rücksicht darauf, dass der Schrifttext das heiligmäßige Leben möglichst vollständig repräsentiere: swaz ie geschach dem lobesamen, I daz leite er unde schreip dar an (774 f.). Als später der Vater des Alexius, Eufemian mit Namen, den Toten findet, da hält dieser den brief, das Schriftstück, in siner bant (944), und zwar derart besigelt und bebaft, dass der Vater ihn mit aller [s]îner kraft | nie mobte drûz gewinnen (973 ff.). Erst als die herbeigeholten großen Herren, die Spitzen von regnum und sacerdotium den heiligen Leichnam anbeten (982 ff.) und ihn bitten, er möge den rodel [Rotulus] und daz brievelîn (996) dem bäbest vrôn als dem vater aller kristenheit (990 E.) aushändigen, auf daz man gehere und ouch gelese, I waz dar an geschriben wese (999 f.), lässt Gott ein zeichen (1002) geschehen. Die beilic hant (1004) öffnet sich, der Papst nähert sich ihr dêmüeteclichen (1008), entnimmt das Schriftstück und übergibt es dem Stiftsschreiber Ethio, der den Text voll-

40 Vgl. besonders auch Strunk: Kunst, S. 74, 81 f., 114 ff., der zugleich den systematischen Ort der für legendarisches Erzählen gattungstypischen Inspirationsbitten genau als die kulturell verfügbare Lösungsmöglichkeit dieser Aporie bestimmt (bes. S. 85 ff.). Systematisch erhellend zu dem hier angesprochenen Problemzusammenhang Luhmann / Fuchs: Beobachtung.

41 Und nicht anders als jene ,Vie de saint Alexis, mit wclcher man die französische Literaturgeschichte beginnen lässt. Die strukturell entscheidenden Interpretamente der folgenden Überlegungen können daher auch für diesen älteren Text cum grano salis Geltung beanspruchen.

42 Alexius ist also auctor und scriptor zugleich, in seinem Schreibakt kommt es zu einer Koppelung zweier Funktionen, welche im Mittelalter alles andere als von vorneherein selbstverständlich oder auch nur üblich ist - vgl. etwa Minnis: Theory; Saenger: Space, S. 249 ff.; Andersen u.a. (Hg.): Autor; Saenger: Lesen, hier S. 189 ff., 206 ff.; Zimmermann (Hg.): mauctork. 
ständig verliest und so den Umstehenden kund tut, dass der Bettler, der ein halbes Leben lang mitten unter ihnen gehaust hatte, der einzige Sohn der Familie gewesen war.

Mit diesem Motiv der eigenhändigen Lebensbeschreibung des Alexius konstruiert die Legende einerseits eine mythopoetische Erzählung davon, dass der Ursprung der legendarischen Tradition des Wissens von ihrem Protagonisten bei diesem selbst liegt. Die Narratologik der AlexiusErzählung ist so angelegt, dass der Ursprung des hagiographischen Wissens ein zunächst autobiographisches Wissen des Heiligen selbst ist. Was die Legende von Alexius erzählt, ist das, was Alexius selbst erzählt hatte. Doch ist dies noch nicht alles. Die epische Konstruktion ist komplexer und sie enthält insbesondere auch ein magisches Element, das sie vor allem deswegen benötigt, weil, wenn man die Kategorien genau nimmt, selbst noch die autobiographische Erzählung selbstverständlich eine Repräsentation ist - und das heißt im Falle der Autobiographie eines Heiligen: eine Repräsentation dessen, was gerade nicht narrativ repräsentiert werden kann, nämlich die Heiligkeit des Heiligen.

Darum tritt zum Moment der Autobiographie das Moment der Autographie ${ }^{43}$ hinzu - ein Moment, das sodann eine Verbindung zwischen der körperlichen Präsenz des Heiligen in der Reliquie seines heiligen Leichnams einerseits und andererseits seiner textuellen Repräsentation in der legendarischen Erzählung herstellen wird. Diese Verbindung, so hoffe ich zeigen zu können, soll die kategoriale Differenz zum Verschwinden bringen, die zwischen dem erzählerischen Text als einem lesbaren Repräsentationssystem einerseits und auf der anderen Seite dem Körper des Heiligen besteht - jenem Körper, welcher als Reliquie nicht allein Objekt von Bedeutungszuschreibungen ist, sondern zugleich Gegenstand ritueller Körper-Praxis, von kultischen Berührungen nämlich, in denen seine thaumaturge Heil(s)-Kraft so auf den Berührenden übergehen kann, dass dieser etwa seiner körperlichen Gebrechen ledig wird (1310 ff.).

Dem epischen Prozess ist dies in der Weise eingeschrieben, dass der autobiographische Text des Heiligen sursprünglich gespeichert ist in einer autographen Schrift. Mit seiner eigenen heiligen Hand schreibt Alexius seine Vita auf, und dieser Schreibakt stiftet eine ontische Identität, einen substanziellen Zusammenhang zwischen dem sakralen Körper und der Schrift des Heiligen dergestalt, dass diese selbst als - im Wortsinne - heilige Schrift ausgezeichnet ist. Diese die Schrift heiligende substanzielle Identität mit dem sakralen Körper zeigt sich als erstem dem Vater Eufemian, als er vergeblich versucht, dem Leichnam seines Sohnes den brief

43 Zur mittelalterlichen Autographie generell vgl. Chiesa / Pinclli (Hg.): Autografi ; Ganz: Mind; Saenger: Space, S. 249 ff.; Quast: Hand-Werk, v.a. S. 74 f. 
aus der Hand zu nehmen. Dieser im Augenblick des Todes des Heiljgen zur Reliquie transformierte Leichnam und das Schriftstück sind untrennbar eins. Die Schrift ist also unmittelbar integraler Teil der wichtigsten Reliquie des heiligen Alexius, und sie hat auch nach der späteren Ablösung von diesem corpus incorruptum selbst Reliquienstatus. ${ }^{44}$ Die Schrift ist hier ein magisches Ding, in welchem das Transzendente vermittlungslos totale Präsenz gewinnt. Sie ist mehrı als nur eine Repräsentation des Heils, sie ist nicht ১bloß< ein differenzieller Speicher für das differenzielle Bedeutungssystem des (autobiographischen) Textes. Es kommt zunächst nicht auf die in der Schrift gespeicherten Bedeutungen an, sondern auf ihre dingliche Materialität, ${ }^{45}$ und deswegen bedarf es, damit die Schrift überhaupt in einem hermeneutischen Sinn gelesen werden kann, allererst ihrer Ablösung vom sakralen Leichnam (998 ff.). Ich verstehe dies als eine narrative Ausdrucksform dafür, dass die Schrift in ihrer Materialität hier auf die Seite der (magischen) Präsenz transzendenten Heils gehört, der gegenüber jeder hermeneutische Akt der Lektüre jedenfalls defizitär bliebe, die vielmehr eine Bearbeitung in den Formen kultischer Unmittelbarkeit erfordert.

Wenn man so interpretieren muss, was Konrads Alexius-Legende über die Identität von sakralem Körper und geheiligter Schrift etzählt, dann ist es freilich nicht mehr überraschend, dass die Herstellung der >Bedeutungshaltigkeitc der Schrift einen Übergang von magischer Gegenwärtigsetzung der Transzendenz auf die Seite repräsentationeller Darstellung erfordert. Oder vielleicht sollte man, weil solche Lesbarkeit die Materialität des Schriftstücks und damit seine reliquiäre Funktionalität ja nicht zum Verschwinden bringt, sondern sozusagen überlagert, genauer sagen: Erforderlich ist die Öffnung der Schrift auf eine neue, auf die Dimension der Bedeutung hin. Eine solche Öffnung - Ent-Schließung heißt es in Konrads Legende: entslozzen wart sîn beilic bant (1004) - ist allerdings ihrerseits prekär: Das Heilige entzieht sich ja gerade der Repräsentierbarkeit. Der Leichnam des Alexius gibt die Schrift daher nicht ohne weiteres aus der Hand. Als von ihm abgelöste könnte sie nicht allein als Reliquie kultisch verwendet, sondern zugleich auch als Text gelesen, als Objekt von Bedeutungszuweisungen verwendet werden. Die Ablösung der Hand-Schrift von der heiligen Hand gestaltet sich daher aufwendig. Die Öffnung der Sphäre transzendenter Unverfügbarkeit des in der Reliquien-Schrift anwesenden Heils in das Reich der Text-Bedeutungen hinein muss ihrerseits unverfügbar gehalten, also selbst sakralisiert werden. Dass sich die heilige

44 Als Sekundärreliquie, vgl. Angenendt: Art. Reliquien, hier S. 69.

45 Vgl. auch Quast: Hand-Werk. 
Hand der Reliquie öffnet, qualifiziert die Erzählung daher einerseits als ein Wunder -

dô wart ein zeichen dâ gesehen

$\mathrm{daz}$ got in allen tet bekant.

(1002 f.) -,

andererseits schiebt sich die heilige Hand des Papstes (990) zwischen den sakralen Leib des Alexius und den ersten Leser des Textes Ethio. ${ }^{46}$ Der primäre Tradent des Schriftstücks kann nicht schon der leibliche, sondem darf erst der geistliche Vater sein: der mit der sakramentalen Amtsgewalt ausgestattete Stellvertreter Gottes auf Erden. ${ }^{47} \mathrm{Im}$ Einssein mit der Schreibhand des heiligen Leichnams wird der Status des Schriftstücks als Reliquie von seiner skripturalen Materialität dominiert. Seine Öffnung auf einen Status, in welchem die kommunikable Bedeutungsqualität des Schriftstücks entscheidend wird, jener Prozess also, in welchem gewissermaßen aus der Materialität der Schrift die Lesbarkeit des Textes hervorgeht, dies wird als Übergabe der Schrift von der heiligen Hand des Verfassers an den Vorleser Ethio etzählt, und der Tradent dieser allerersten traditio ist die Instanz sakraler Tradition selbst. Es sind also die thaumaturge Schreibhand des Alexius und die gesalbte Hand des Papstes, welche die Schrift als Bedeutungsträger freigeben, welche unterscheiden, unter wie gearteten Bedingungen für wen die Schrift in welchen Dimensionen als Träger von Bedeutungen lesbar sein kann oder darf, und unter welchen Unständen nicht.

In dem Augenblick, wo das Schriftstück nicht allein mehr durch seine substanzielle Einheit mit der Reliquie des Heiligen bestimmt ist, sondern zugleich ein Text wird und Prozessen der Entzifferung unterzogen werden kann, in dem Moment also, wo sich - wir wissen: wie bei jedem Text - Bedeutungsfragen stellen können, ${ }^{48}$ wird die ontologische Sicherung der autographen Schriftreliquie ergänzt um die saktamentale und traditionale Sicherung des autobiographischen Schrifttextes. Dieser wird in den kirch-

46 Die Gesamtüberlieferung der Alexius-Tradition wird an dieser Stelle, also über die Frage, wer der erste Tradent des Alexius-Autographen ist, in einen so genannten bräutlichen und einen späpstlichen Zweig geteilt, von denen überdies ein dritter unterschieden werden könnte, in welchem sowohl der Papst als auch die Kaiser die Schrift erhalten; vgl. hierzu Löffler: Alexius, S. 3 et pass. Zur weiteren Forschung vgl. die I.iteraturangaben bei Storey: Bibliography, S. 11 ff., 53 ff., 61 ff; zur lateinischen und mittclhochdeutschen Tradition überdies Rosenfeld: Art. ^Alexiuss; Löffler: Alexius, zumal dic Zusammenfassungen S. $115 \mathrm{ff}$, $240 \mathrm{ff}$.

47 Kur Theiligkeit des Papstes vgl. Fuhrmann: IHeiligkeits; Bagliani: I.eib.

48 Fragen mithin, auf welche nur strittige Antworten möglich sind. Ausnahmen gibt cs allein im Rahmen substanzialistischer Bedeutungskonzeptionen (vgl. unten), und die promincntesten Beispiele für derartige Ausnahmen sind - so versteht sich - heilige und magische Texte: Offenbarungen, Zaubersprüche usw. 
lichen Tradierungs- und Traditionsraum integriert und gleichsam erneut eingeschlossen in eine hochexklusive und wenn nicht direkt liturgische, so doch wenigstens besonders liturgienahe Kommunikationssituation. In Gegenwart des heiligen Leichnams selbst, in Anwesenheit des Papstes als der Verkörperung sakramentaler Amtsgewalt und im Beisein der Verwandten entnimmt (so könnte der Text formulieren) der Stiftsschreiber also der Verwalter der sakralen Schrifttraditionen - der Schriftreliquie einen Text, indem er die Vita des Heiligen entziffert; weist er also (so müssten wir sagen) der Schrift, sie verlesend, Bedeutung zu. Mit dieser Mythopoetik des Legendarischen wird sozusagen die chronologische, systematische und axiologische Sekundarität jeder Heiligenerzählung gegenüber ihrem Erzählgegenstand unterlaufen, indem das Erzählen als ein Element der materialen Hinterlassenschaft, als Reliquie des Heiligen selbst konstituiert ist.

Die hier interessierende epische Konstruktion der Alexius-Legende erzählt eine Geschichte von der Lesbarmachung der Schrift. Sie erzählt gewissermaßen sich selbst, sie lässt die hagiographische Erzählung als einen Träger von Bedeutungen über den autobiographischen Text und über die Sekundärreliquie der autographen Schrift aus der Primärreliquie des sakralen Leibes des toten Heiligen selbst hervorgehen. Und das heißt: Die Bedeutungen der Hagiographie führen zurück auf und gehen hervor aus einer Sphäre der Unlesbarkeit, in welcher die Schrift und der Körper ontisch ungeschieden, in welcher sie substanziell identisch sind. In dieser Sphäre, wo sich transzendentes Heil unmittelbar in der Immanenz manifestiert, ist die Schrift gerade nicht als ein Träger von Bedeutungen bedeutsam, sondern als Träger dessen, was von allen Bedeutungs- als Differenzordnungen kategorial unterschieden ist: als Träger transzendenten Heils. Und aus dieser Sphäre wird die Schrift in die Ordnung der Lesbarkeit verschoben eben in einem gestuften Prozess der Einführung von Differenzierungen: (1) die Unterscheidung von Körper und Schriftträger, die der Papst vollzieht, indem er den Rotulus aus der Hand des heiligen Leichnams entnimmt und an den Schreiber weiterreicht; (2) die Unterscheidung von Schriftträger und Schrift im Akt der Lektüre durch den Stiftsschreiber; (3) die Unterscheidung von Schrift und Text, die in der Dimension der mündlichen Verlautbarung des Textes manifest witd.

Hinzuzufügen ist freilich, dass diese Unterscheidungen erzählt werden, damit sie gerade nicht bemerkbar sind: Die mythopoetische Dimension der Erzählung liegt ja offenkundig darin, dass sie eine Traditionsgeschichte erzählt, dass sie im Modus der traditio gerade den traditionalen Zusammenhalt von Körper und Schrift und Text behauptet. Indem sie sie aus einer Schrift hervorgehen lässt, deren reliquiäre Bedeutsamkeit nicht aus Bedeutungen und damit aus Bedeutungsdifferenzen stammt, sucht die 
Erzählung die Bedeutungen des Textes mit Höchstgeltung zu versehen und zu vereindeutigen: TTextbedeutunge wird zum Singular. Sie kann etscheinen nicht als Moment pluraler Bedeutungszuweisungen, sondern als gewissermaßen ontischer Sachverhalt, als sein vermittlungsloses /Wesen', das der Text durch seine traditio-Geschichte hindurch bewahrt. Fundierend also ist für Konrads Erzählung ein historisch weithin fremd gewordenes, ein substanzialistisches Konzept, welches Bedeutung unmittelbar aus dem Bedeuteten hervorgehen sieht: hier in der Dimension des Schriftträgers durch die wundersame Auflösung der Einheit von heiliger Hand und Rotulus, auf der Ebene der Schrift als Autograph und auf derjenigen des Textes als Autobiographie. Polysemie oder Polyvalenz als Textmerkmal sind durch ein solches Konzept ebenso ausgeschlossen, wie die darauf beruhende Möglichkeit der Strittigkeit von Bedeutungszuweisungen.

4.

Die aristokratischen Laienkulturen des 12. und 13. Jahrhunderts kennen freilich nicht allein Geschichten, die von der Produktion der Lesbarkeit von Schrift erzählen, davon also, wie eine Schrift auf Bedeutungen hin geöffnet werden kann, welche ihrerseits Dimensionen der Unlesbarkeit besitzt und qua dieser nicht nur substanziell sbedeutungshaitig, sondern zugleich magisch wirkmächtig ist. Es gibt vielmehr auch den sozusagen komplementären Fall: Das Erzählen davon, wie die Schrift von den Bedeutungen sozusagen wieder Abstand nimmt - sie transzendiert -, um nicht als Medium stets semiotisch vermittelter Bedeutungen, sondern als Träger unvermittelten, vermittlungslos skriptural präsenten Heils zu funktionieren. Notwendig muss es sich dabei um einen Prozess handeln, in welchem all jene Unterscheidungen swieder sistiert werden, vermittels welcher im Falle der Alexius-Legende aus dem sakralen Leichnam schrittweise fromme Bedeutungen entbunden worden waren.

Driu liet von der maget nennt man (im Blick auf A 4870 f. $4^{\circ}$ ) die älteste episch ausgebreitete deutschsprachige Mariendichtung, die ein priester Wern$h e r^{50}$ auf Veranlassung eines wohl gleichfalls dem Kreis der Augsburger Weltkleriker zuzuordnenden Manegolt 51 verfasste. Der allein fragmentarisch

49 Zitate und Versangaben (unter Verwendung der jeweiligen Handschriftensiglen) nach der Ausgabe: Wernher: Maria. Zum - wenig eindrucksvollen - aktuellen Stand der Forschung zu diesem Text vgl. Gärtner: Art. Priester Wernher. Den Singular iText gebrauche ich im Folgenden als Sammelausdruck für die verschicdenen handschriftlichen Versionen des variablen Überlieferungsfeldes.

50 C 1296 / A 1136 / D 1242, C 5802 / A 4813.

51 C 5812 / A 4822. 
und in Überarbeitungen überlieferte Text datiert sich selbst auf das Jahr 1172,52 wobei er zugleich auf die Geschichte von sacerdotium und regnum Bezug nimmt, ${ }^{53}$ und er wist deutlich gegliedert in drei durch Prologe eingeleitete liet, ,Bücher, die drei hohen kirchlichen Festen zugeordnet sind: das erste Mariä Geburt (8. Sept.), das zweite Mariä Verkündigung (25. März), das dritte der Geburt Jesu aus Maria (Weihnachten und den unmittelbar anschließenden Festen der Weihnachtszeit bis Lichtmeß)«.54 Diese heilsgeschichtliche Zuordnung ist inhaltlich bestimmt, und dementsprechend wird im Kern folgendes erzählt - in den Worten eines nochmals die Integrität des Textes sichernden epilogischen Rückblicks der Bearbeitung A:

daz aine liet saget

von der kumfte Marien, der edelen $v \bar{n}$ der freyen, wie ir múter sei gewan bey ainem hailigen man der Joachim genant ist. das ander liet zclet die frist, daz si wart gemahelot als der heilant gebot,

- also Marias Vermählung mit dem greisen Joseph sowie die Verkündigung -

daz dritte daz si den gebar

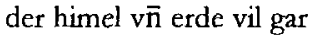

gemachet hat mit sinem list

$v \bar{n}$ allez daz darinne ist,

bede groz vñ chlaine

[...].

(A 4872-4885)

Dieses epische Gerüst wird aufgefüllt unter anderem mit Episoden aus der Kindheit Marias und ihrem Leben im Tempel; mit dem Gertenmirakel, durch welches sie Joseph zugesprochen wird; mit dessen Bestürzung über ihre Schwangerschaft; endlich mit einem Schlussteil, der auf das Leben Jesu ausblickt, auf seine Passion, Höllenfahrt, Auferstehung und Himmelfahrt sowie schließlich das Jüngste Gericht.

Erzählt ist dies auf der Grundlage des (wie man heute sagen muss: apokryphen Pseudo-) Matthäus-Evangeliums ${ }^{55}$ : Aus dieser ihrer hebräischen Gefangenschaft ${ }^{56}$ habe allerdings, so Wernhers Text, der heilige Hierony-

52 C $5799 \mathrm{ff}$. / A $4809 \mathrm{ff}$.

531172 ist das 13. Jahr des 1159 mit der Papstwahl Alexanders III. beginnenden Schismas (A $4851 \mathrm{ff}$ / D 5111) und das 22. (recte: das 20.) Jahr der Kaiserherrschaft von Friedrich I. Barbarossa (A $4866 \mathrm{f}$ ), in welchem Jahr dieser Polen unterwarf (A $4862 \mathrm{f}$ ).

54 Gärtner: Art. Priester Wernher, Sp. 907.

55 Vgl. Gärtner: Art. Priester Wernher, Sp. $909 \mathrm{ff}$.

56 vgl. G 87 / AD 87 ff. 
mus die rede befreit in die senfte lateine (AD 93). ${ }^{57}$ Dieserart Wasser in Wein und Milch in Öl verwandelnd (AD 94 f.), habe Hieronymus die schlafende schriff58 geweckt, ${ }^{59}$ so dass sie für gläubige Zuhörer in geradezu eucharistieanaloger Weise zu heilbringendem Wein, brôt unt [...] spîse $e^{60}$ werden konnte. ${ }^{61} \mathrm{Als}$ dritter in dieser Tradenten-Kette nun habe Wernher mit diutischer rede daz buoch ${ }^{62}$ verfertigt. ${ }^{63}$

Dabei wird der Zweck dieses Übergangs von der lingua sacra in die Sprache der Alltagskommunikation, dieses merklichen Verzichts ${ }^{64}$ also auch auf die Geltungsfonds der heiligen Sprache und ihrer sakralen institutionellen Orte, deutlich angegeben. Es geschieht dies, auf

daz si [das buoch] alle muosen lesen

die gotes chint wellen wesen,

unt ouch mugen schouwen

laien unde frouwen [A: phaffen, layen, vrowen],

smechen [als geistliche Speise, P. S.] unt ergrunden

von dem frônen chinde,

daz im die muoter erchôs

57 Wiederum liegt also ein substanzialistisches Bedeutungskonzept zugrunde. Es muss hier gegenüber der Bedeutung der Rede das sprachliche Medium (Hebräisch - Latein sodann auch Deutsch) als zugleich akzidenziell und essenziell auffassen. Oder anders: Die Sprachgestalt in der lingua sacra ist der heiligen Rede gegenüber zugleich notwendig und kontingent. Hier zeigt sich einer der Fundamentalwidersprüche substanzialistischer Bedeutungskonzeptionen.

58 G 104 / A 102 / D 105.

59 Zu derartigen Metaphoriken vgl. Spitz: Metaphorik, hier etwa S. 142 ff., 158 ff.

60 GD $108 \mathrm{f}$. / A $106 \mathrm{f}$.

61 Zur rgeistlichen Speiser vgl. Lange: „Geistliche Speise»; Gellrich: Idea; Ohly: Wirkungen, besonders S. $44 \mathrm{ff}$. In schöner Prägnanz fordert etwa der ,Prologus` zur so genannten ,Wenzelsbibek seine Leser und Hörer auf: Esset und trincket mein lieben früt von der Heiligen Schrift Und werdet truncken nv czu stunt (zitiert nach: Wenzelsbibel, fol. $1^{\mathrm{va}}$ ); vgl. hierzu Mentzel-Reuters: "Oufsliessen deiner schrifte tor", hier S. 200 f. - Zu fragen wäre, ob nicht eine Ausdehnung einer Poetologie der Eucharistie-Analogie auf einen zwar geistlichen, nicht aber mehr kanonisch sakrosankten Text, wie sie an Wernhers Mariengedicht zu beobachten ist, ob sie nicht ein Streiflicht wirft auf jene diskursiven Verhältnisse, die etwa der Eucharistie-Diskurs in Gotfrits von Straßburg ,Tristan (dazu zuletzt Kellner: Eigengeschichte, besonders S. 164 ff.) als eine seiner Möglichkeitsbedingungen voraussetzt.

62 GF 139 f. / A $137 \mathrm{f}$. / D $139 \mathrm{f}$.

63 Wernher rangiert damit gewissermaßen auf einer Ebene mit dem Evangelisten Matthäus und dem Kirchenvater Hieronymus. nVor der humanistischen Übersetzergeneration des 15. Jahrhunderts dürfte solch eine kulturgeschichtliche Dimensionierung des Übersetzens in die Volkssprache singulär sein« (Henkel: Erzählen, S. 5).

64 Er wird freilich abgemildert dadurch, dass lateinische Einsprengsel in den mittelhochdeutschen Text dessen Herkunft aus der lingua sacra auch im Verlauf des epischen Prozessen gegenwärtig halten. 
diu ir magetuom nie verlôs

unt niemer mac verliesen.

[GFD 141-149/ A 139-145]

Ausdrücklich also bestimmt sich Wernhers Marienerzählung als Text für ein breites (alle), auch weibliches und vor allem für ein laikales Publikum. ${ }^{65}$ Dies impliziert zugleich, dass die epische Vergegenwärtigung des Lebens der Gottesmutter hier einen gewissen Abstand gewinnt gegenüber kultisch-rituell strukturierten Aufführungssituationen. ${ }^{60}$ Erst unter dieser Prämisse laikal dominierter Kommunikationskreise allerdings sind nun auch jene funktionalen Selbstbeschreibungen ernsthaft diskutierbar, auf welche ich hier hinaus will und welche sich vor allem in einer Art von Binnenepilog zum zweiten liet finden.

Der Rahmen dieses Funktionskonzeptes zunächst ist einer der zentralen typologisch-heilsgeschichtlichen (und misogynen) Erzähl- und Bedeutungszusammenhänge, ${ }^{67}$ in welchen christliche Überlieferung den systematischen Nexus von Sündenfall und Erlösung unter anderem ausfaltet und mit welchem Wernhers Erzählung auch anhebt. Sie handelt

65 Vgl. auch F 186 / A 174, C 2961 f. / A 2497 f., C 3049 ff. / A 2557 ff., C 5743 / A 4755. In C $5707 \mathrm{ff}$ / A $4719 \mathrm{ff}$. (ir wuoteriche [...] enmuget daz himelriche ersturmen mit den schilten!) ist überdies näherhin auf die adelige Gewaltfähigkeit von Zuhörern des Textes angespielt. - Zum Publikum auch Bumke: Mäzene, S. 231 f.; Fromm: Art. Wernher, hier S. 264.

66 Indizien der handschriftlichen Textüberlieferung stützen übrigens diese Annahme. Dies gilt insbesondere im Falle der Handschrift Krakau, Bibl. Jagiellonska, Berol. mgo 109 (früher Berlin, Preußische Staatsbibliothek, Ms. germ. oct. 109). Sie überliefert die wohl noch Ende des 12. Jahrhunderts und also gleichzeitig mit dem neuen höfischen Roman entstandene Bearbeitung D (vgl. Gärtner: Art. Priester Wernher, Sp. 913), und ist eine der besonders prominenten Epenhandschriften des frühen 13. Jahrhunderts: "Mit diesem Werk besitzt D[eutschland] in Europa die reichhaltigste Quelle zur Ikonogr[aphie] des M[arienlebens] unmittelbar $\mathrm{n}$ [ach] 1200, am Anfang der erst aufblühenden M[arien]verehrungw. (Nitz: Art. Marienleben, Zitat Sp. 230) Am einfachsten zugänglich ist diese Denkmal über die 1925 erschiencne Ausgabe: Des Priesters Wernher drei Lieder von der Magd. Nach der Fassung der Handschrift der Preußischen Staatsbibliothek metrisch übersetzt und mit ihren Bildern hg. von Hermann Degering. (Auswahlreihe des Volksverbandes der Bücherfreunde) Berlin o.J. Die Bilder gehören in den stilistischen Zusammenhang der Regensburger Buchmalerei des 12. Jahrhunderts; vgl. Klemm: Regensburger Buchmalerei; Henkel: Bild und Text. Die Handschrift insgesamt ist vom "Typ her ein adeliger Literaturpflege zugedachtes $A$ ndachts- und Erbauungsbuch. [...] Dass adeliger Kunstwille hinter der Handschrift steht, scheint $[\ldots]$ sicher. Als Auftraggeber für die Handschrift müsste man den in Scheyern residierenden Hof der Wittelsbacher unter Ludwig II. denken, in dessen Umgebung auch die der gleichen Zeit entstammende illuminierte Handschrift von Veldekes IEneasroman anzusiedeln ist" (Henkel: Erzählen, S. 8).

67 Der jüngste Übersichtsartikel hierzu stammt von Suntrup: Heilsgeschichts-Konzeptc. 
von ir diu allen wiben

den itewîz hât benomen,

daz der tôt was bechomen

von dem êrsten wibe in die werlt.

$[\ldots]$

wie wol siz allez undervie

swaz Eva sunden begie.

[CAD 8-11, $19 \mathrm{f}$.]

Mit und durch Maria kommt jene Erlösung in die Welt, die durch den paradiesischen Lapsus erforderlich geworden ist. Dabei ist der heilsgeschichtliche Bezug Eva - Maria ${ }^{68}$ nicht nur durch die Funktionskomplementarität von Sündenfall und Erlösung, sondern zugleich auch durch die beiden gemeinsame Mutter-Funktion gestiftet: Eva ist mater [...] cunctorum viventium (Gen. 3,20), Maria ist Gottesmutter. Und auch an dieser MutterFunktion selbst kann der typologische Sinnzusammenhang aufgewiesen werden. Mit dem Sündenfall kommt in doppelter Weise Selbstreferenz in die Welt, nämlich als Scham ${ }^{69}$ und als Schmerz. Gott bestraft den Sündenfall auch mit der Selbstwahrnehmung des menschlichen Körpers, darunter an erster Stelle der Geburtsschmerz: mulieri quoque dixit: multiplicabo aerumnas tuas et conceptus tuos; in dolore paries filios (Gen. 3,16). Dass dieserart die Schmerzhaftigkeit des Gebärens auf die Rolle Evas beim Sündenfall zurückverweist, sie gewissermaßen bei jedem einzelnen Geburtsvorgang neu körperlich präsent macht und insofern eine Form des Gedächtnisses ist, dies braucht als elementare kulturelle Selbstverständlichkeit nicht je wieder artikuliert zu werden, um doch in jedem alteuropäischen Diskurs über den Schmerz gegenwärtig sein zu können.

Umgekehrt ist Erlösung als Schmerzfreiheit codierbar. Es ist daher ein zentrales Theologoumenon, dass Maria Jesus nicht nur im Zustand der Virginität, sondern auch ohne Wehen gebiert; die Geburt des Heilands serinnert den Sündenfall auch insofern sozusagen kontrafaktisch. In Wernhers Marienerzählung lautet die entsprechende Passage:

diu geburt tet ir niht wê

[D: wan sie in âne meil enpfie

$\overrightarrow{\mathrm{v}}$ ane sunde gebar

von rehte ir leides nien wâr.]

$[\ldots]$

daz grôze wuofen unde chlagen,

daz ander frouwen muozen haben,

sô si gewinnent diu kint

68 Vgl. zur Übersicht Söll: Art. Eva-Maria-Parallele, S. 420 f.

69 Gen. 3,6 f: et tulit de fructu illius et comedit, deditque viro suo, qui comedit, et aperti sunt oculi amborum. cumque cognovissent esse se nudos, consuerunt folia ficus et fecerunt sibi perizomata. 
diu von sunden chomen sint,

daz was ir seltsâne;

si was sîn alles âne.

[D: wan daz kint daz da furgie,

do siz cem ersten enpfie,

daz brûefte der heilige geist

ane suntliche uolleist.

uon schulden sie niht enswar,

do sie Christum gebar,

$(\ldots)]$

[C 4097, 4119-4124 / D 3871-3874, 3891-3902 / A 3365, 3387-3392] $]^{70}$

Die Erzählung von Maria und der Erlösung ist implizite stets zugleich eine Erzählung von Eva und dem Sündenfall sowie davon, wie mit dem aus der 'Erbsünder resultierenden Schmerz der je eigene Körper als wahrgenommener in die Welt kommt und wie er dadurch, dass Gott in Maria einen Körper annimmt, wie er also vermittels der Inkarnation des göttlichen Logos wieder verlassen werden kann.

Im Rahmen dieses alle christliche Weltkonstitution zentrierenden heilsgeschichtlichen Deutungsgefüges formuliert nun Wernhers Marienerzählung einen bemerkenswerten Bewirkungszusammenhang ihrer selbst, beansprucht sie eine hochspezifische Leistungsfähigkeit. Es handelt sich dabei um einen Anspruch, der in der deutschsprachigen Literatur des 12. und 13. Jahrhunderts außergewöhnlich bleibt und der allerdings auf kategorialen Entdifferenzierungen beruht, welche in dieser Hinsicht seinen Abstand zu den Prinzipien der Lesbarkeit vergrößern. Der Text beansprucht nämlich Bedeutsamkeit auch gewissermaßen jenseits seiner Textualität und der ihr zugeordneten Bedeutungen, und zwar: magische Bedeutsamkeit. ${ }^{71}$ Wernhers Erzählung - doch setzt dieser Ausdruck immer schon sinnbildende Differenzierungen voraus, die an dieser Stelle gerade aufgehoben sind -, der Codex und seine Schrift und sein Text also werden nicht allein in der Erzählkommunikation gute Dienste leisten, indem sie nämlich das Wissen von Maria, von ihrer heilsgeschichtlichen Leistung, ihrer ethischen Virtuosität und dem darin aktualisierten göttlichen Gesetz kulturell verfügbar zu halten gestatten, sondern auch in einer in gewissem Sinne nichtkommunikativen Praxis - als thaumaturger Geburtshelfer:

70 Fragmente dieser Passage sind überdies in neuaufgefundenen Makulaturstreifen von eincr Handschrift aus dem zweiten Viertel des 13. Jahrhunderts überliefert: Pichler / Reichert: Fragmente, hier S. 209. Vgi. auch A $2523 \mathrm{ff}$.

71 Vgl. oben Anm. 6 sowie speziell Spangenberg: Maria, S. $63 \mathrm{ff}$. 
Swelich weip dise driv liet hat, so si ze keminaten ${ }^{72}$ gat, in ir zesewen hant bevangen, si leidet niht lange weipliche sorgen: daz kint, daz a verborgen waz in ir leibe, daz kvmet in kvrzer weile von ir genedichlichen. div vnkraft můz entweichen $v \bar{n}$ alle sere bose, div Even getelose in dise werlde gesant, do si gotes niht erkannt, do si v $\bar{n}$ ir man wurden vngehorsam.

[A 2505-2520/D 2853-2866] ${ }^{73}$

Wie die Engel an Stelle von Hebammen (C 4099 / A 3366: obstetricum vice) Maria bei der schmerzfreien Heilandsgeburt beistanden, so kann auch das Buch benutzt werden, das jene Geschichte erzählt. ${ }^{74}$ Man kann also den Schrift-Codex verwenden wie eine magisch heilkräftige Reliquie, und wie

72 Kemenate ist unter anderem der weibliche Rückzugsraum, in dem hier die Geburt stattfindet; vgl. auch Strohschneider: Kemenate, hier S. 29 ff.

73 Das Fragment $C^{2}$ überliefert nur einen Teil der zitierten Verse: C 2973-2980. - Die Stelle ist in der Forschung als Ausnahmebeleg für samuletthafte[n] Gebrauch (Henkel: Religiöses Erzählen, S. 5 Anm. 18) religiöser Literatur beschrieben; vgl, auch Wachinger: Gesundheit, S. 18.

74 Und wie die für die Mutter schmerzfreie Geburt Christi diesen nicht in seiner menschlichen Gestalt, sondern als göttlichen Erlöser ausweist, so gewährleistet auch der Codex-Schrift-Text nicht nur eine schmerzfreie Geburt, sondern zugleich die somatische und spirituelle Gesundheit - also Erlösungsfähigkeit - des Neugeborenen:

hie schult ir wizzen under diu:

swâ disiu buochel alliu driu

$<$ werdent behalten>,

diu maget wil des walten,

daz dâ nehein kint

werde krump noch blint,

$<$ noch $>$ niemer werde geborn

daz êwiclíche sî verlorn,

sine welle ez selbe fristen

zuo dem jungisten,

so der lîp mit manigem sêre

scheidet von der sêle.

[C 3027-3038 / A 2539-2550 / D 2867-2876]

In der Sache noch einmal wiederholt wird dies im Epilog der Version A $4898 \mathrm{ff}$. 
jene kann man daher auch diesen teilen ${ }^{75}$, ohne zugleich der in ihm anwesenden Kraft verlustig zu gehen:

swelich wip reine

niht hât wan daz eine [der drei Bücher, P. S.],

des ist zwivel nehein,

si geniezze < sin > etlîch teil,

sô ez ir gât an die nôt:

die maget vliuhet der tôt.

[C 3039-3044 /A 2551-2556]

Der Tod und, wie man ergänzen darf, gleichermaßen die Sünde und der Schmerz fliehen die heilige Jungfrau. Wo sie gegenwärtig ist, können jene nicht sein. Präsent aber ist sie in Text, Schrift und Codex von Wernhers Mariengedicht. ${ }^{76}$ Allein darauf kann nämlich dessen praktische somatische Heilkraft bei Schwangerschaft und Geburt und Neugeborenen beruhen. Buch und Schrift sind im vorliegenden Falle ja keine acheiropoizte 77 oder Kontaktreliquie, sie gehen nicht - wie die vera icon der heiligen Veronika oder die Auto(hagio)graphie des Alexius - materialiter und kontagiös aus dem Moment einer körperlichen Anwesenheit von Transzendenz in der Immanenz der Welt hervor und ihre magische Bedeutsamkeit kann daher auch nicht in einer Herkunftsgeschichte narrativ entfaltet werden. So bedeutsam und bedeutungsvoll aufgeladen die Geschichte der rede, des Textes, ist (Matthäus - Hieronymus - Wernher), so profan stellt sich ja die Geschichte von Schrift und Codex in diesem Falle dar: Manegolt hat den Pfaffen Wernher so lange in seinem Haus beherbergt, bis dieser im Jahre 1172 div geistlîchen liet (C 5820) fertig geschrieben hatte (C 5799 ff. / A $4809 \mathrm{ff}$.), und jede fromme Frau kann die Schrift kopieren, um sie weiter zirkulieren zu lassen. ${ }^{78}$ Die beanspruchte Heilkraft bezieht Wernhers Marienerzählung also nicht aus der Geschichte von Buch oder Schrift, sondern von dem her, was wir den Erzählgegenstand nennen müssten. Nicht die Farbpigmente der getrockneten Tinte oder die Kuhhaut in ihrer per-

75 Anders scheint Ernst: Sprachmagie, S. 156, die hier interessierenden Textstellen zu verstehen. - Zur Reliquienteilung vgl. Dobschütz: Art. Reliquien, hier Sp. 538; Angenendt: Corpus incorruptum, hier S. 332 ff.; Angenendt: Ehre der Altäre, hier S. 237 ff.; Angenendt: Heilige, S. 148 ff.; Legner: Reliquien, S. 6 f.

76 Deswegen können überdies die Bewohner jedes Hauses, in swelhem [...] diu scbrift gelat (C 3045), geistlicher Freude und Seligkeit (C 3046: mandunge) gewiß sein, und deswegen auch ist es zugleich ein Auftrag Gottes und seiner Mutter, das Buch zu kopieren und es zu senden $[\ldots]$ ze minne in dem umberinge verre ynde nâben, also für seine weitere Verbreitung zu sorgen (C 3049-3058 / A 2557-2566).

77 Vgl. Dobschütz: Christusbilder; Belting: Bild, besonders S. 64 ff. et pass.

78 Vgl. Anm. 76. 
gamentenen Dinglichkeit: Maria selbst ist die Geburtshelferin. ${ }^{79}$ Sie wird offenbar unmittelbar körperlich berührt von einer Frau, die dise driv liet bat $[\ldots]$ in ir zesewen bant bevangen (A 2505-2507). ${ }^{80}$

Maria, so wird man zu sagen möglicherweise nicht umhin kommen, kann in bestimmten Verwendungszusammenhängen von Wernhers Gedicht auf eine gewisse Weise in dessen Schriftgestalt substanziell anwesend sein. Dieserart aber setzen die Wirksamkeitsansprüche des Textes ein Konzept voraus, das nicht allein zwischen der Schrift und dem Schriftträger, dem zum Codex gefügten Pergament, keine Unterscheidung vorzunehmen erlaubt, sondern ebenso wenig zwischen der Schrift und dem Text sowie zwischen dem Text und demjenigen, worauf er referiert: der so geschichtlichen wie heilsgeschichtlichen Realität der Gottesmutter. Schriftträger, Schrift, narrativer Text und Erzählgegenstand sind im Zusammenhang gynäko-magischer Praxis zwar völlig gleichnotwendig, doch ununterscheidbar ${ }^{81}$ - und als solcherart ununterscheidbare ist die Schrift dann auch unlesbar. Im Schriftkörper, so beansprucht es Wernhers Text, wirkt eine zugleich körperliche und transzendente Kraft. Diese kann man wohl nur (und kann man zugleich nicht) als Effekt einer irgendwie gearteten (und sei es auch gegenüber der >Realpräsenz` Christi in der Eucharistie sozusagen abgestuften) Gegenwärtigsetzung Marias im Codex denken. Die Mysterien der Religion kehren begriffssprachlich als Paradoxien wieder: Der Schriftkörper und der heilige Leib der Gottesmutter stehen in einem

79 Der Epilog der Bearbeitung A expliziert die Motivation der Gottesmutter für die Schwangerschaftshilfe und damit den heilsgeschichtlichen Deutungsrahmen des magischen Funktionsmechanismus. Maria steht der Schwangeren gegen den Strafspruch Gottes (Gen. 3,16 - vgl. oben bei Anm. 69) hilfreich bei in Erinnerung an die thr selbst bei der Geburt Christi gewährte Schmerzfreiheit:

ir [ $=$ der Schwangeren] weget div kuniginne

vor gotes antwurte,

$v \bar{n}$ gedenchet ir geburtte [= Geburt Christi]

div ir wart ertailet.

(A 4894-4897)

Zur Geburtshelferschaft Marias vgl. in frömmigkeitsgeschichtlicher Perspektive Schreiner: Maria, bes. S. $57 \mathrm{ff}$.

80 Systematisch eng Verwandtes diskutiert Boureau: Franciscan Piety, hier S. 13 ff. - Das hier rekonstruierte magische Konzept des Codex ralsc Maria kehrt, an jenes vielleicht anschließend, ein Modell in gewisser Weise um, nach welchem Maria allegorisch als Buch aufgefasst werden kann. In der deutschen Literatur $m$. W. am ausführlichsten begegnet solche Auslegung bei Heinrich von St. Gallen (Hilg: Marienlebenc, hier S. 132 ff. [Kap. II]); vgl. dazu Kesting: Maria; Schreiner, »... wie Maria geleicht einem puchu.

$81 \mathrm{Vgl}$ hierzu Czerwinski: Gegenwärtigkeit, bes. auch S. 12 ff. - Zu den zahlreichen Gemeinsamkeiten wie zu den theoretischen und methodischen Differenzen zwischen Czerwinskis Konzept und den von mir verfolgten Ansätzen vgl. Strohschneider: Zeichen; sowie Czerwinski: Alterität. 
Verhältnis von ontischer Identität und Nicht-Identität. Diese Form der Präsenz (die keine ist) der Gottesmutter ist nicht durch hermeneutische Sinnzuschreibungen zu behandeln, sie ist nicht lesbar. Man kann sich ihr aber im Schriftkörper haptisch ebenso versichern, wie in den zahllosen magischen Kultbildern und -statuen der abendländischen Marienfrömmigkeit. Für diese Auflösung kategorialer Differenzierungen, die unter den Rationalitätsprämissen wissenschaftlicher Diskurse gerade nicht hintergehbar sind oder überschritten werden können, wird hier der Ausdruck imagisch verwendet: Nicht überhaupt, nicht also etwa beim Schreiben, Vorlesen, Lesen oder Hören von Wernhers Marienleben oder beim Betrachten der handschriftlichen Illuminationen, wohl aber im Gebrauch als Instrument praktischer Geburtshilfe operiert der Codex-Schrift-Text keineswegs unter den hier wiederholt angeführten Grundgesetzen allen Zeichenhandelns. In diesem Gebrauch ist er zwar nicht unabhängig von aller Semantik, aber eben auch nicht wegen ihr somatisch wirk- und bedeutsam. Hier ist die Schrift nicht ein gegenwärtiges Mittel der Verweisung auf Abwesendes, sondern selbst ein Ort, an welchem sich heilige Präsenz manifestiert: eine magische Gegenwärtigkeit des transzendenten Heils in der Welt. Dass in diesem Sinne Maria im Codex-Schrift-Text gegenwärtig sei, dies lässt daher keineswegs den Umkehrschluss zu, sie sei dann anderswo, etwa bei Gott, abwesend: Sie unterliegt gerade nicht der Disjunktion von Anwesenheit und Absenz, und das heißt, Marias Anwesenheit im thaumaturgen Codex ist eine 'Anwesenheit, die "Anwesenheit" nicht genannt werden kann - aber >Abwesenheits ebenso wenig.

5.

Im Wechsel vom hermeneutischen Gebrauch des vom Priester Wernher verfassten Schrifttextes zu jener somatisch heilkräftigen Verwendung des Schriftkörpers, in welcher dieser wie eine Reliquie funktioniert, die qua Berührung mit dem Heiland, seiner Mutter oder einem Heiligen transzendentes Heil und magische Heilkraft gewissermaßen speichert und beides im körperlichen Kontakt (in der haptischen Berührung, im Kuss, in der Einverleibung) weitergeben kann, ohne sich dabei zu verbrauchen: In diesem Gebrauchswechsel ist eine Grenze überschritten. Es ist dieselbe Grenze, über welche hinweg - sozusagen in umgekehrter Richtung - der autograph-autobiographische Schrift-Text des Alexius tradiert wird, wenn dieser Schrift-Text auf die gebetsförmige Anrufung des Heiligen hin von der Hand der sakralen Reliquie (mit welcher er substanziell verbunden war) freigegeben und von der Hand des Gottesstellvertreters an einen Schreiber zur ,Vokalisierung weitergereicht wird. Beide hier vorgestellten 
Texte - der Konrads von Würzburg ebenso wie derjenige des Augsburger Weltklerikers ein Jahrhundert zuvor - unterscheiden mithin eine Praxis der Zuweisung differenzieller Bedeutungen, eine Praxis der Lektüre, in welcher Schrift als Medium semiotischer Stellvertretung und repräsentatorischer Darstellungen fungiert, von einem Gebrauch, in welchem der Schrift gerade in einer bedeutungsfernen Dimension Bedeutsamkeit zukommt: als Ort der Manifestation einer vermittlungslosen Gegenwärtigkeit transzendenten Heils. In solcher Bedeutsamkeit öffnet sich eine Sphäre jenseits der Unterscheidung von Anwesenheit und Abwesenheit, jenseits der Disjunktion von Bezeichnendem und Bezeichnetem, mithin jenseits aller Lesbarkeiten. Es handelt sich um eine Sphäre heiliger Entdifferenzierungen in jenem Sinne, in welchem man - für die Fans von George Spencer Brown: sehr formal - heilig dasjenige nennen könnte, was von allen Unterscheidungen durch einen Unterschied getrennt ist. ${ }^{82}$ In dieser Sphäre entfallen Unterschiede, die sonst kategorial unhintergehbar sind, wie diejenigen von Körper oder Schriftträger und Schrift, von Schrift und Text, schließlich insbesondere eben auch die im Begriff des Zeichens gefasste Einheit der Unterscheidung von Bedeutetem und Bedeutendem.

Die Alexius-Legende wie die Marienerzählung unterscheiden allerdings nicht allein zwei Schriftpraxen und mit diesen eine Ordnung der Differenz (mithin der Zeichen, der Bedeutungen, der Kommunikation) von einer Sphäre der Nicht-Differenz. Sie unterstellen auch - oder: sie formulieren ein kulturelles Wissen erheblicher historischer Relevanz davon, dass selbst die Schriftgestalt von Texten die dieserart errichtete Grenze passieren kann. Dieses kulturelle Wissen verbindet Skripturalität keineswegs ausschließlich mit dem Begriff der Lesbarkeit und also mit demjenigen der Abwesenheit. Nach ihm kann Schrift nicht allein als Träger von Bedeutungen funktionieren, sondern auch zum Beispiel als Träger transzendenten Heils. Sie ist dann nicht Gegenstand hermeneutischer Operationen, sondern Dingobjekt einer nicht-hermeneutischen Praxis, die von der Lesbarkeit der Schrift in bestimmten Hinsichten durchaus abzusehen imstande ist. Diese Gebrauchsform versichert sich durch körperliche Berührung einer Anwesenheit des Abwesenden, und zwar einer Präsenz, welche für sie jedenfalls in dem Sinne reak ist, dass das Heil und die Heilkraft des in diesen Fällen: transzendenten - Absenten unmittelbar praktisch wirksam werden kann. Hierauf viel eher als auf ihrer Lesbarkeit kann die Hochstbedeutsamkeit von Schrift beruhen in einer Praxis, die Semiotisches und Somatisches in sehr fremd gewordener Weise konfiguriert. Dic Alterität einer solchen Weltkonstruktion mag man sich also auch daran

$82 \mathrm{Vgl}$. Luhmann: Ausdifferenzicrung, I.uhmann: (jesellschaft, S. $230 \mathrm{ff}$;, Luhmann: Religion, Luhmann / Fuchs: Beobachtung; Strohschneider: Inzest-I leiligkeit. 
vergegenwärtigen, dass für sie - anders als unser alltäglicher Gebrauch des komplexen Wortes `Bedeutung es nahe legt - einerseits die Bedeutsamkeit und andererseits die Lesbarkeit selbst eines Sachverhaltes wie der Schrift nicht direkt proportional sind. Man könnte auch sagen: Hermeneutik und Relevanz von Schrift verhalten sich hier durchaus spannungsvoll zueinander.

Konfigurationen, wie ich sie hier - mühsam genug - zu beschreiben versuche, sind auf den Feldern der Schrift im Verlauf der spätmittelalterlichen und neuzeitlichen Verschriftlichungsschübe allmählich in residuale Sektoren der Kultur abgedrängt worden. Völlig verschwunden sind sie nicht. Die Geltungssicherung von Texten durch die eigenhändige Unterschrift, welche einen abwesenden Körper wergegenwärtigt in der Spur seiner (seit der Erfindung des Buchdrucks: einzigartigen) Bewegung ${ }^{83}$, die Speicherung von symbolischem wie ökonomischem Kapital in Autographensammlungen (oder Autogrammkarten), ${ }^{84}$ Verfahren der konkreten-visuellen Poesie ${ }^{85}$, die Praxis von Tätowierungen ${ }^{86}$ : Dies und anderes mehr zehrt noch immer in der einen oder anderen Weise von nicht-repräsentatorischen Dimensionen von Schrift. Zumindest setzt es solche Dimensionen der Unlesbarkeit doch voraus - wie dies auch noch die dumpfen Versuche der Auslöschung von Bedeutung durch die Verbrennung von Büchern tun. ${ }^{87}$

Alles andere als möglicherweise residual ist freilich, wenn man den Bereich der Schrift verlässt, selbst in zeitgenössischen Kulturen der Bedarf an Unterscheidungen zwischen den Prozessen der Bedeutungszuweisung und solchen Praxen, in denen Sachverhalte gerade in ihrer Unlesbarkeit Bedeutsamkeit besitzen. Jede Ausübung körperlicher Gewalt oder die Oper als synästhetisches Ereignis oder der Sport oder die Techno-Perfor-

83 Vgl. Hahn: Handschrift, S. 205 f.

84 Eincr der großen Autographensammler der deutschen Literaturgeschichte, Goethe, hat in einem Bricf an Jacobi vom 10. Mai 1812 wic folgt formuliert, was hier gemeint ist: »denn da mir die sinnliche Anschauung durchaus unentbehrlich ist, so werden mir vorzügliche Menschen durch ihre Hanúschrift auf eine magische Weise vergegenwärtigtw. (Goethe: Werke, II, 7, S. 58) Zum Sammeln von Autographen vgl. etwa Mecklenburg: Autographensammeln; Ganz: Mind, S. 298 f.; Krevert / Kammeier (Hg.): Autographen.

85 Vgl. Adler / Iirnst: 'Text als Figur.

$86 \mathrm{Vgl}$. oben $\Lambda \mathrm{nm} .26$.

87 Vgl. Iöwenthal: Calibans Erbe; Rafetseder: Bücherverbrennungen.; Verweyen: Bücherverbrennungen; für das Mittelalter Werner: Vernichtet und Vergessen? - Übrigens vollzicht dic am 10. Mai 1933 verwendete Formel der Barbarei sIch übergebe den Flammen die Schriften von ... gewalttätig genau jene Entdifferenzierung, auf wclche es mir ankommt: Thr Ausdruck dic Schriftenc unterscheidet gerade nicht zwischen Skripturalität und Textualität und Semantik. 
mances der Berliner Loveparade, um es bei diesen Beispielen hier zu belassen: Sie bieten hinlänglich auch aktuelles Anschauungsmaterial für Handlungszusammenhänge, bei welchen es für diejenigen, die in sie involviert sind, entscheidend ankommen kann auf die Regeln der Unterscheidung dessen, was als Lesbares, und dessen, was in seiner Unlesbarkeit behandelt werden muss.

\section{Bibliographie}

Adler, Jeremy / Ulrich Ernst: 'Text als Figur. Visuelle Poesie von der Antike bis zur Moderne. Weinheim 1987.

Andersen, Elizabeth / Jens Haustein / Anne Simon / Peter Strohschneider (Hg.): Autor und Autorschaft im Mittelalter. Kolloquium Meißen 1995. Tübingen 1998.

Angenendt, Arnold: Corpus incorruptum. Eine Leitidee der mittelalterlichen Reliquienverehrung. In: Saeculum 42 (1991), S. 320-348.

Angenendt, Arnold: Heilige und Reliquien. Die Geschichte ihres Kultes vom frühen Christentum bis zur Gegenwart. München 1994.

Angenendt, Arnold: Zur Ehre der Altäre erhoben. Zugleich ein Beitrag zur Reliquienteilung. In: Römische Quartalschrift für christliche Altertumskunde und Kirchengeschichte 89 (1994), S. 221-244.

Angenendt, Arnold: Art. Reliquien / Reliquienverchrung. II. Im Christentum. In: Theologische Realenzyklopädic 29 (1998), S. 69-74.

Assmann, Alcida: Die Sprache der Dinge. Der lange Blick und die wilde Semiose. In: Ians Ulrich Gumbrecht / K. Ludwig Pfeiffer (Ig): Materialität der Kommunikation. Frankfurt/M. 1988, S. 237-251.

Assmann, Aleida / Jan Assmann: Art. Schrift I. In: Historisches Wörtcrbuch der Philosophie 8 (1992), Sp. 1417-1429.

Assmann, Jan: Herrschaft und Heil. Politische Theologie in Ägypten, Israel und Luropa München, Wien 2000.

Assmann, Jan: Religion und kulturelles (jedächtnis. \%ehn Studien. München 2000.

Aust, Iugo: Art. Iesen. In: Reallexikon der deutschen literaturwissenschaft 2 (2000), \$. 406-410.

Bagliani, Agostino Paravicini: Der Leib des Papstes. Kine Theologic der Ilinfalligkeit. Aus dem Italienischen übersetzt von Ansgar Wildermann. München 1997

Barwise, John: Situationen und klcine Welten. In: Arnim Stechow / Dieter Wunderlich (Ig.): Semantik. Win internationales Handbuch der zeitgenössischen forschung. Berlin. New York 1991, S. 80-89.

Belting, Hans: Bild und Kult. Fine (ieschichte des Bildes vor dem \%eitalter der Kunst. Munchen ${ }^{2} 1991$

Belting, Jans: Bild-Anthropologie. Fintwüfe für eine Bildwissenschaft. München 2001.

Bet\%. Jiohannes]: Art. "iucharistice. In: Heinrich Firies (Hg.): Handbuch theologischer (irundbegriffe. 4 Bdc. München ${ }^{2} 1973$, Bd. 1, S. 371-391.

Blumenberg, Hans: Arbeit am Mythos. Irankfurt/M. ${ }^{51990 .}$

Blumenberg, I Ians: I ic I esbarkeit der Welt. Frankfurt/M. 1986.

Boureau, Nlain: Franciscan Picty and Voracity. Uses and Strategems in the Hagiographic Pamphlet. In: Roger (harticr (IIg): The Culture of I'rint. P'ower and the Uses of P'rint in Early Modern Europe. Cambridge 1989, S. 13.59. 
Bühler, Karl: Sprachtheorie. Die Darstellungsfunktion der Sprache. Mit einem Geleitwort von Friedrich Kainz. [Ungekürzter Nachdruck der Ausgabe Jena 1934] Stuttgart, New York 1982.

Bumke, Joachim: Mäzene im Mittelalter. Die Gönner und Auftraggeber der höfischen Literatur in Deutschland 1150-1300. München 1979.

Campe, Rüdiger: Art. Lecture. In: Reallexikon der deutschen Literaturwissenschaft 2 (2000), S. 385-388.

Cancik, Hubert: Art. Ikonoklasmus. In: Handbuch religionswissenschaftlicher Grundbegriffe 3 (1993), S. 217-221.

Chiesa, Paolo / Lucia Pinelli (Hg.): Gli autografi medievali. Problemi paleografici e filologici. Spoleto 1994.

Classen, Albrecht $(\mathrm{Hg}$ ): The Book and the Magic of Reading in the Middle Ages. New York, London 1998.

Costamagna, Giorgio: Kryptographie. In: Hartmut Günther / Otto Ludwig (Hg.): Schrift und Schriftlichkeit. Ein interdisziplinäres Handbuch internationaler Forschung. 2 Halbbde. Berlin, New York 1994/1996, S. 1608-1616.

Cresswell, M. J.: Die Weltsituation. In: Arnim Stechow / Dieter Wunderlich (Hg): Semantik. Ein internationales Handbuch der zeitgenössischen Forschung. Berlin, New York 1991, S. 71-80.

Czerwinski, Peter: Der Glanz der Abstraktion. Frühe Formen von Reflexivität im Mittelalter. Exempel einer Geschichte der Wahrnehmung I. Frankfurt/M., New York 1989.

Czerwinski, Peter: Gegenwärtigkeit. Simultane Räume und zyklische Zeiten, Formen von Regeneration und Genealogie im Mittelalter. Exempel einer Geschichte der Wahrnehmung II. München 1993.

Czerwinski, Peter: Alterität oder bistoria non fecit saltus. Peter Czerwinski antwortet Peter Strohschneider. In: Internationales Archiv für Sozialgeschichte der deutschen Literatur 21, 1 (1996), S. 166-180.

Czerwinski, Peter: Verdichtete Schrift / comprebensiva scriptura. Prolegomena zu einer Theorie der Initiale. In: Internationales Archiv für Sozialgeschichte der deutschen Literatur 22, 2 (1997), S. 1-35.

(Zerwinski, Peter: per visibilia ad invisibilia. 'Texte und Bilder vor dem Zeitalter von Kunst und Literatur. In: Internationales Archiv für Sozialgeschichte der deutschen Literatur $25,1(2000)$, S. 1-94.

Denzinger, Henricus : Enchiridion Symbolorum, Definitionum et Declarationum de rebus fidei et morum. [...] edidit Carolus Rahner. Barcelona, Freiburg/Br., Rom ${ }^{31} 1957$.

Didi-Huberman, Georges: Vor einem Bild. Aus dem Französischen von Reinold Werner. München, Wien 2000.

Dobschütz, Irnst von: Christusbilder. Untersuchungen zur christlichen Legende. Leipzig 1899.

1) obschütz, Renate: Art. Reliquien. In: Lexikon der christlichen Ikonographic 3 (1971), Sp. 538-546.

Iigidi, Margreth u.a. (Hg.): (iestik. Figuren des Körpers in Text und Bild. Tübingen 2000.

Vinst, Ulrich: Sprachmagie in fiktionaler I iteratur. Textstrukturen - Zeichenfelder - Theoriescgmente. In: Arcadia 30 (1995), S. 113-185.

Virnst, Ulrich: fiormen der Schriftlichkeit im höfischen Roman des hohen und späten Mittelalters. In: Fiühmittelalterliche Studien 31 (1997), S. 252-369.

Virnst, Wolfgang: $\Lambda$ rt. Absenz. In: Asthetische (irundbegriffe 1 (2000), S. 1-16.

leistner, Fdith: Historische Typologie der deutschen Heiligenlegende des Mittelalters von der Mitte des 12. Jahrhunderts bis zur Reformation. Wiesbaden 1995.

field, Helmut: Das Verstandnis des Abendmahls. Darmstadt 1976. 
Fromm, Hans: Art. Wernher (Priester oder Pfaffe?) - Verfasser eines mittelhochdeutschen Marienlebens. In: Literatur Lexikon. Autoren und Werke deutscher Sprache 12 (1992), S. 263-264.

Fuhrmann, Horst: Die Heiligkeitr des Papstes. In: H. F.: Einladung ins Mittelalter. München 1987, S. 151-168.

Gärtner, Kurt: Art. Priester Wernher. In: Verfasserlexikon 10 (1999), Sp. 903-915.

Ganz, David: ,Mind in character: Ancient and Medieval Ideas about the Status of the Autograph as an Expression of Personality. In: P. R. Robinson / Rivkah Zim (Hg.): Of the Making of Books. Medieval Manuscripts, their Scribes and Readers (Festschrift Malcolm Beckwith Parkes). Aldershot 1997, S. 280-299.

Ganz, Peter ( $\mathrm{Hg}$ ): Das Buch als magisches und als Repräsentationsobjekt. Wiesbaden 1992.

Geier, Manfred: Sekundäre Funktionen von Schrift. In: Hartmut Günther / Otto Ludwig (Hg.): Schrift und Schriftlichkeit. Ein interdisziplinäres Handbuch internationaler Forschung. 2 Halbbde. Berlin, New York 1994/1996, S. 678-686.

Gellrich, Jesse M:: The Idea of the Book in the Middle Ages. Language Theory, Mythology, and Fiction. Ithaca, London 1985.

Gipper, H[elmut]: Art. Bedeutung. In: Historisches Wörterbuch der Philosophie 1 (1971), Sp. 757-759.

Gladigow, Burkhard: Art. Kultbild. In: Handbuch religionswissenschaftlicher Grundbegriffe 4 (1998), S. 9-14.

Goethe, Johann Wolfgang von: Sämtliche Werke, Briefe, Tagebücher und Gespräche. II Abteilung: Briefe, 'Tagebücher und Gespräche. Hg. von Karl Eibl u.a. Bd. 7: Von 1812 bis zu Christianes Tod. Ig. von Rose Unterberger. Frankfurt/M. 1994.

Griese, Sabine: Gebrauchsformen und Gebrauchsräume von Einblattdrucken des 15. und frühen 16. Jahrhunderts. In: Volker Honemann u.a. (Hg.): Einblattdrucke des 15. und frühen 16. Jahrhunderts. Probieme, Perspektiven, Fallstudien. 'Tübingen 2000, S. 179208.

Günther, Hartmut / Otto Ludwig (Hg.): Schrift und Schriftlichkcit. Ein interdisziplinäres Handbuch internationaler Forschung. 2 I Halbbde. Berlin, New York 1994/1996.

Gumbrecht, Hans Ulrich: Das Nicht-Hermencutische. Skizze einer Genealogie. In: Jörg Huber / Alois Martin Müller (Hg.): Dic Wiederkehr des Anderen. Basel, Irankfurt/M. 1996, S. $17-35$.

Gumbrecht, Hans Ulrich: Form ohne Materic versus form als Ercignis. In: Henk de Bern / Matthias l'rangel (Hg.): Systemtheoric und I Iermencutik. 'Tübingen 1997, S. 31-46.

Gumbrecht, I lans Ulrich: 'I'en Brief Reflections on Institutions and Re/Presentations. In (iert Melville (I Ig): Institutionalität und Symbolisierung. Verstetigungen kultureller Ordnungsmuster in Vergangenheit und Gegenwart. Köln, Weimar, Wien 2001, S. 69-75.

(Sumbrecht, Jans Urich / K. I udwig Pfeiffer (Ig): Schrift. München 1993.

I Jaarmann, I larald: Universalgeschichte der Schrift. Frankfurt/M., New York ${ }^{2} 1991$.

Jahn, Alois: Handschrift und Iätowierung. In: Jans Ulrich ( Bumbrecht / K. Iudwig I'feiffer (I Ig.): Schrift. München 1993, S. 201-217.

I Jarms, Wolfgang: In Buchstabenkërpern die Chiffen der Welt lesen. Zur Inszenicruty von Wërtern durch figurale oder verdinglichte Buchstaber. In: Jan-Dirk Müller (Hg.): suffihrunge und sehrifte in Mittelalter und Fruher Neuzcit. Stuttgart, Weimar 1906 , S. $575-595$

I Ieidegger, Martin: Sein und \%cit. 'Tübingen 171903

Ilenkel, Nikolaus: Religiöses Firzählen um 1200 im Kontext höfischer Iitcratur. Pricster Wernher, Konrad von Fußesbrunnen, Konrad von Heimesfurt. In: 'I'imothy R. Jackson 
/ Nigel F. Palmer / Almut Suerbaum (Hg): Die Vermittlung geistlicher Inhalte im deutschen Mittelalter. Internationales Symposium, Roscrea 1994. Tübingen 1996, S. 1-21.

Henkel, Nikolaus: Bild und Text. Die Spruchbänder der ehem. Berliner Handschrift von Priester Wernhers Mariar. In: Scrinium Berolinense. 2 Bde. Hg. von Peter Jörg Becker u.a. Wiesbaden 2000, Bd. 1, S. 246-275.

Hilg, Hardo: Das 'Marienleben des Heinrich von St. Gallen. Text und Untersuchung. Mit einem Verzeichnis deutschsprachiger Prosamarienleben bis etwa 1520. München, Zürich 1981.

Hörisch, Jochen: Brot und Wein. Die Poesie des Abendmahls. Frankfurt/M. 1992.

Hohmeyer, Jürgen: „Bist du Gott, so wehr dich«. In: Der Spiegel 11 (12.3.2001), S. 222 225.

Illich, Ivan: Im Weinberg des Textes. Als das Schriftbild der Moderne entstand. Ein Kommentar zu Hugos "Didascalicon«. Aus dem Englischen von Ylva Erikson-Kuchenbuch. Frankfurt/M. 1991.

Illich, Ivan: Von der Prägung des Er-Innerns durch das Schriftbild. Überlegungen zur >Arche Noahı des Hugo von St. Victor. In: Aleida Assmann / Dietrich Harth ( $\mathrm{Hg}$ ): Mnemosyne. Formen und Funktionen der kulturellen Erinnerung. Frankfurt/M. 1991, S. 48-56.

Iserioh, Erwin: Art. Abendmahl III/2. Mittelalter. In: Theologische Realenzyklopädie 1 (1977), S. 89-106.

Jackson, Timothy R.: Konrad von Würzburg's Legends. Their Historical Context and the Poet's Approach to his Material. In: Peter F. Gan\% / Werner Schröder (Hg.): Probleme mittchochdeutscher Frzähiformen. Marburger Colloquium 1969. Berlin 1972, S. 197-213.

Jackson, Timothy R.: The Legends of Konrad von Würzburg. Form, Content, Function. Firlangen 1983.

Jacger, C. Stephen: The Envy of Angels. Cathedral Schools and Social Ideals in Medieval Europe, 950-1200. Philadelphia 1994.

jacger, C. Stephen: Charismatic Body - Charismatic 'Text. In: Exemplaria 9 (1997), S. 117-137.

Jauß, Hans Robert: Über religiöse und ästhetische Erfahrung. Zur Debatte um I Ians Beltings ,Bild und Kulte und George Stciners ,Von realer Gegenwart. In: Merkur 45, 9/10 (1991), S. 934-946.

Jorissen, Hans: I) ie Intfaltung der Transsubstantiations-Lehre bis zum Beginn der Hochscholastik. Münster 1965.

Kellner, Beate: Figengeschichte und literarischer Kanon. Zu cinigen Formen der Selbstbeschreibung in der volkssprachlich-deutschen literatur des Mittelalters. In: B. K. / Ludger lieb / Peter Strohschncider (Hg.): Litcrarische Kommunikation und soziale Interaktion. Studien zur Institutionalität mittelaiterlicher Literatur. Frankfurt/M. u.a. 2001, S. $153-182$.

Kesting, Peter: Maria als Buch. In: Würzburger Prosastudien I. Wort-, begriffs- und textkundliche Intersuchungen. Hg. von der Forschungsstelle für deutsche Prosa des Mittclalters am Scminar für dcutsche Philologic der Universität Würzburg. München 1968, S. 122-147.

Kieserling, André: Kommunikation unter Anwesenden. Studien über Interaktionssysteme. lirankfurt/M. 1999.

Kippenberg, Jans (;. / Brigitte Luchesi (I Ig.): Magie. Die sozialwissenschaftliche Kontro. verse über das Verstehen fremden I) enkens. Frankfurt/M ${ }^{21995}$ 
Kippenberg, Hans G.: Art. Magie. In: Handbuch religionswissenschaftlicher Grundbegriffe 4 (1998), S. 85-98.

Klemm, Elisabeth: Die Regensburger Buchmalerei des 12. Jahrhunderts. In: Regensburger Buchmalerei. Von frühkarolingischer Zeit bis zum Ausgang des Mittelalters. Ausstellung der Bayerischen Staatsbibliothek München und der Museen der Stadt Regensburg. München 1987, S. 39-58.

Konrad von Würzburg: [Alexius] Die Legenden II. Hg. von Paul Gereke. Halle/S. 1926.

Koschorke, Albrecht: Körperströme und Schriftverkehr. Mediologie des 18. Jahrhunderts. München 1999.

Krevert, Peter / Heinz-Ulrich Kammeier ( $\mathrm{Hg}$ ): Autographen und Autogramme. Eine faszinierende Leidenschaft. Bd. 1. Münster 1999.

Küsters, Urban: Der lebendige Buchstabe. Christliche Traditionen der Körperschrift im Mittelalter. In: Horst Wenzel / Wilfried Seipel / Gotthart Wunberg (Hg.): Audiovisualität vor und nach Gutenberg. Zur Kulturgeschichte der medialen Umbrüche. Wien, Mailand 2001, S. 107-117.

Laarmann, Matthias: Transsubstantiation. Begriffsgeschichtliche Materialien und bibliographische Notizen. In: Archiv für Begriffsgeschichte 41 (1999), S. 119-150.

Lange, Klaus: "Geistliche Speise«. Untersuchungen zur Metaphorik der Bibelhermeneutik. Diss. phil. Kiel 1963.

Legner, Anton: Reliquien in Kunst und Kult zwischen Antike und Aufklärung. Darmstadt 1995.

Leipold, Inge: Die Auftraggeber und Gönner Konrads von Würzburg. Versuch einer 'Theorie der Literatur als soziales Handelnc. Göppingen 1976.

Löffler, Roland: Alexius. Studien zur lateinischen Alexius-Legende und zu den mittelhochdeutschen Alexius-Dichtungen. Diss. phil. Freiburg/Br. 1991.

Löwenthal, Leo: Calibans Erbe. Bücherverbrennungen und kulturelle Verdrängungsmechanismen. In: Aleida Assmann / Jan Assmann ( $\mathrm{Hg}$.): Kanon und Zensur. München 1987, S. 227-236.

Luhmann, Niklas: Einfache Sozialsysteme. In: N. L.: Soziologische Aufkłärung 2. Aufsätże zur Theorie der Gesellschaft. Opladen 1975, S. 21-39.

Luhmann, Niklas: Die Ausdifferenzierung der Religion. In: N. L.: Gesellschaftsstruktur und Semantik. Studien zur Wissenssoziologie der modernen Gesellschaft. Bd. 3. Frankfurt/M. 1989, S. 259-357.

Luhmann, Niklas / Peter Fuchs: Von der Beobachtung des Unbeobachtbaren. Ist Mystik ein Fall von Inkommunikabilität? In: N. L. / P. F.: Reden und Schweigen. Frankfurt/M. 1989, S. 70-100.

Luhmann, Niklas: Die Form der Schrift. In: Hans Ulrich Gumbrecht / K. Ludwig Pfeiffer (Hg.): Schrift. München 1993, S. 349-366.

Luhmann, Niklas: Die Kunst der Gesellschaft. Frankfurt/M. 1995.

Luhmann, Niklas: Die Gesellschaft der Gesellschaft. 2 Bde. Frankfurt/M. 1997.

Luhmann, Niklas: Die Religion der Gesellschaft. Hg. von André Kieserling. Frankfurt/M. 2000.

Lyons, John: Bedeutungstheorien. In: Arnim Stechow / Dieter Wunderlich (Hg.): Semantik. Ein internationales Handbuch der zeitgenössischen Forschung. Berlin, New York 1991, S. 1-24.

Macho, Thomas: Bemerkungen zu einer philosophischen 'Theorie der Magic. In: Ilans Peter Duerr ( Ig.): Der Wissenschaftler und das Irrationale. 1. Bd.: Beiträge aus Ethnologie und Anthropologie. Frankfurt/M. 1981, S. 330-350.

Mazzoni, Ira: Die blinden $\Lambda$ ugen des Bildersturms. In: Süddeutsche Zeitung vom 15. März 2001, S. 17. 
Mecklenburg, Günther: Vom Autographensammeln. Versuch einer Darstellung seines Wesens und seiner Geschichte im deutschen Sprachgebiet. Marburg 1963.

Meggle, Georg / Geo Siegwart: Der Streit um Bedeutungstheorien. In: Marcelo Dascal / Dietfried Gerhardus / Kuno Lorenz / G. M. (Hg.): Sprachphilosophie. Ein internationales Handbuch zeitgenössischer Forschung. 2. Halbbd. Berlin, New York 1996, S. 964-989.

Mentzel-Reuters, Arno: „Oufsliessen deiner schrifte tork. Mitteldeutscher Biblizismus und die Wenzelsbibel. In: Joachim Heinzle / L. Peter Johnson / Gisela Vollmann-Profe (Hg.): Literatur im Umkreis des Prager Hofs der Luxemburger. Schweinfurter Kolloquium 1992. Berlin 1994, S. 174-206.

Minnis, Alastair J.: Medieval Theory of Authorship. Scholastic Literary Attitudes in the Later Middle Ages. Aldershot ${ }^{2} 1988$.

Mölk, Ulrich: La Chanson de saint Alexisc et le culte du saint en France aux XIe et XII siècles. In: Cahiers de Civilisation Médiévale 21 (1978), S. 339-355.

Neef, Christian: Besudelung im Namen Allahs. In: Der Spicgel 10 (5.3.2001), S. 170.

Neumann, Gerhard / Sigrid Weigel (Hg.): Die Lesbarkeit der Kultur. Literaturwissenschaften zwischen Kulturtechnik und Ethnographie. München 2000.

Neunheuser, Burkhard: Eucharistie in Mittelaltex und Neuzeit. Freiburg, Basel, Wien 1963.

Nitz, Michael: Art. Marienleben. In: Lexikon der christlichen Ikonographie 3 (1971), Sp. 212-233.

Oettermann, Stephan: Zeichen auf der Haut. Die Geschichte der Tätowierung in Europa. Frankfurt/M. 1985.

Ohly, Friedrich: Ausgewählte und neue Schriften zur Literaturgeschichte und Bedeutungsforschung. Hg. von Uwe Ruberg und Dietmar Peil. Stuttgart, Leipzig 1995.

Ohly, Friedrich: Wirkungen von Dichtung. In: Deutsche Vierteljahrsschrift für Literaturwissenschaft und Geistesgeschichte 67 (1993), S. 26-76.

Ottmann, Henning: Politische Theologie als Begriffsgeschichte. Oder: Wie man die politischen Begriffe der Neuzeit politisch-theologisch erklären kann. In: Volker Gerhardt (Hg.): Der Begriff der Politik. Bedingungen und Gründe politischen Handelns. Stuttgart 1990, S. 169-188

Otto, Rudolf: Das Heilige. Über das Irrationale in der Idee des Göttlichen und sein Verhältnis zum Rationalen. [Nachdruck der ungekürzten Sonderausgabe 1979] München 1997.

Palmer, Nigel F.: Eine Prosabearbeitung der Alexiuslegende Konrads von Würzburg. In: Zeitschrift für deutsches Altertum und deutsche Literatur 108 (1979), S. 158-180.

Pichler, Gerd / Hermann Reichert: Neue Fragmente von Priester Wernhers Mariar. In: Zeitschrift für deutsches Altertum und deutsche Iiteratur 125 (1996), S. 202-210.

Quast, Bruno: Hand-Werk. Die Dinglichkeit des Textes bei Konrad von Heimesfurt. In: Beiträge zur Geschichte der deutschen Sprache und Literatur 123 (2001), S. 65-77.

Rafetseder, Hermann: Bücherverbrennungen. Die öffentliche Hinrichtung von Schriften im historischen Wandel. Wien u.a. 1988.

Rehberg, Karl Siegbert: Weltrepräsentanz und Verkörperung. Institutionelle Analyse und Symboltheorien. Eine Einführung in systematischer Absicht. In: Gert Melville $(\mathbf{H g}$ ): Institutionalität und Symbolisierung. Verstetigungen kultureller Ordnungsmuster in Vergangenheit und Gegenwart. Köln, Weimar, Wien 2001, S. 3-49.

Rosenfeld, Hans-Friedrich: Art. AAlexius, In: Verfasserlexikon 1 (1978), Sp. 226-235

Rothacker, Erich: Probleme der Kulturanthropologie. Bonn 1965 [41988].

Saenger, Paul: Space Between Words. The Origins of Silent Reading. Stanford 1997. 
Saenger, Paul: Lesen im Spätmittelalter. In: Roger Chartier / Guglielmo Cavallo (Hg.): Die Welt des Lesens. Von der Schriftrolle zum Bildschirm. Frankfurt/M., New York 1999, S. 181-217.

Schaefer, Ursula: Vokalität. Altenglische Dichtung zwischen Mündlichkeit und Schriftlichkeit. Tübingen 1992.

Schlaffer, Heinz: Der Umgang mit Literatur. Diesseits und jenseits der Lektüre. In: Poetica 31 (1999), S. 1-25.

Schreiner, Klaus: »... wie Maria geleicht einem puch«. Beiträge zur Buchmetaphorik des hohen und späten Mittelalters. In: Archiv für Geschichte des Buchwesens 11 (1971), Sp. 1437-1464.

Schreiner, Klaus: Maria. Jungfrau, Mutter, Herrscherin. München, Wien 1994.

Schreiner, Klaus: Buchstabensymbolik, Bibelorakel, Schriftmagie. Religiöse Bedeutung und lebensweltiche Funktion heiliger Schriften im Mittelalter und in der Frühen Neuzeit. In: Horst Wenzel / Wilfried Seipel / Gotthart Wunberg (Hg.): Die Verschriftlichung der Welt. Bild, Text und Zahl in der Kultur des Mittelalters und der Frühen Neuzeit. Wien, Mailand 2000, S. 59-103.

Schreiner, Klaus: Das Buch im Nacken. Bücher und Buchstaben als zeichenhafte Kommunikationsmedien in rituellen Handlungen der mittelalterlichen Kirche. In: Horst Wenzel / Wilfried Seipel / Gotthart Wunberg (Hg.): Audiovisualität vor und nach Gutenberg. Zur Kulturgeschichte der medialen Umbrüche. Wien, Mailand 2001, S. 72-95.

Schreiner, Klaus: Heilige Buchstaben, Texte und Bücher, die schützen, heilen und helfen. Formen und Funktionen mittelalterlicher Schriftmagie. In: Erika Greber / Konrad Ehlich / Jan-Dirk Müller (Hg.): Materialität und Medialität von Schrift. Bielefeld 2002, S. 73-89.

Sckommodau, Hans: Alexius in Liturgie, Malerei und Dichtung. In: Zeitschrift für romanische Philologie 72 (1956), S. 165-194.

Söll, Georg: Art. Eva-Maria-Parallele. In: Marienlexikon 2 (1989), S. 420 f.

Sontag, Susan: Against Interpretation [1964]. In: S. S.: Against Interpretation and Other Essays. Toronto, New York 1966, S. 3-14.

Spangenberg, Peter-Michael: Maria ist immer und überall. Die Alltagswelten des spätmittelalterlichen Mirakels. Frankfurt/M. 1987.

Spitz, Hans-Jörg: Die Metaphorik des geistigen Schriftsinns. Ein Beitrag zur allegorischen Bibelauslegung des ersten christlichen Jahrtausends. München 1972.

Spree, Axel: Kritik der Interpretation. Analytische Untersuchungen zu interpretationskritischen Literaturtheorien. Paderborn [u.a.] 1995.

Steiner, George: Von realer Gegenwart. Hat unser Sprechen Inhalt? Mit einem Nachwort von Botho Strauß. Aus dem Englischen von Jörg Trobitius. München, Wien 1990.

Storey, Christopher: An Annotated Bibliography and Guide to Alexis Studies (La vie de Saint Alexis). Genève 1987.

Strohschneider, Peter: Die Zeichen der Mediävistik. Ein Diskussionsbeitrag zum Mittclalter-Entwurf in Peter Czerwinskis ‘Gegenwärtigkeit،. In: Internationales Archiv für Sozialgeschichte der dcutschen Literatur 20, 2 (1995), S. 173-191.

Strohschneider, Peter: Situationen des Textes. Okkasionelle Bemerkungen zur $>$ New Philologyc. In: Zeitschrift für deutsche Philologie 116 (1997), Sonderheft: Philologie als Textwissenschaft. Alte und neue Horizonte, S. 62-86.

Strohschneider, Peter: Inzest-Heiligkeit. Krise und Aufhebung der Unterschiede in Hartmanns x Gregoriust In: Christoph Huber / Burghart Wachinger / Hans-Joachim Ziegeler (Hg.): Geistliches in weltlicher, Weltliches in geistlicher Literatur des Mittclalters. Tübingen 2000, S. 105-133. 
Strohschneider, Peter: Kemenate. Geheimnisse höfischer Frauenräume bei Ulrich von dem Türlin und Konrad von Würzburg. In: Jan Hirschbiegel / Werner Paravicini (Hg.): Das Frauenzimmer. Die Frau bei Hofe in Spätmittelalter und früher Neuzeit. 6. Symposium der Residenzen-Kommission der Akademie der Wissenschaften in Göttingen. Stuttgart 2000, S. 29-45.

Strohschneider, Peter: Textheiligung. Geltungsstrategien legendarischen Erzählens im Mittelalter am Beispiel von Konrads von Würzburg >Alexiust. In: Gert Melville / Hans Vorländer ( $\mathrm{Hg}$ ): Geltungsgeschichten. Über die Stabilisierung und Legitimierung institutioneller Ordnungen. Köln, Weimar, Wien 2002.

Strunk, Gerhard: Kunst und Glaube in der lateinischen Heiligenlegende. Zu ihrem Selbstverständnis in den Prologen. München 1970.

Suntrup, Rudolf: Typologische Heilsgeschichts-Konzepte in mittelalterlicher geistlicher Literatur. In: Volker Honemann / Tomas Tomasek (Hg.): Germanistische Mediävistik. Münster, Hamburg, London ${ }^{2} 2000$, S. 277-309.

Thürnau, Donatus: Art. Bedeutung. In: Reallexikon der deutschen Literaturwissenschaft 1 (1997), S. 204-207.

Verweyen, Theodor: Bücherverbrennungen. Eine Vorlesung aus Anlass des 65. Jahrestages der $\gg$ Aktion wider den undeutschen Geist $\ll$. Heidelberg 2000.

sVie de saint Alexis،. Das Leben des heiligen Alexius. Aus dem Altfranzösischen übersetzt von Klaus Berns. München 1968.

Wachinger, Burghart: Erzählen für die Gesundheit. Diätetik und Literatur im Mittelalter. Heidelberg 2001.

Warnke, Martin (Hg): Bildersturm. Die Zerstörung des Kunstwerks. München 1973.

Wellberry, David: Die Äußerlichkeit der Schrift. In: Hans Ulrich Gumbrecht / K. Ludwig Pfeiffer (Hg.): Schrift. München 1993, S. 337-348.

Wenzel, Horst: Partizipation und Mimesis. Die Lesbarkeit der Körper am Hof und in der höfischen Literatur. In: Hans Ulrich Gumbrecht / K. Ludwig Pfeiffer (Hg.): Materialität der Kommunikation. Frankfurt/M. 1988, S. 178-202.

Wenzel, Horst: Hören und Sehen, Schrift und Bild. Kultur und Gedächtnis im Mittelalter. München 1995.

Wenzel, Horst / Christina Lechtermann: Repräsentation und Kinästhetik. In: Paragrana 10, 1 (2001), S. 191-213.

Wenzelsbibel. König Wenzels Prachthandschrift der deutschen Bibel. Exläutert von Horst Appuhn. Mit einer Einführung von Manfred Kramer. 8 Bde. Dortmund 1990.

Werner, Thomas: Vernichtet und Vergessen? Bücherverbrennungen im Mittelalter. In: Otto Gerhard Oexle (Hg.): Memoria als Kultur. Göttingen 1995, S. 149-184.

Wernher, Priester: Maria. Bruchstücke und Umarbeitungen. Hg. von Carl Wesle. 2. Aufl. besorgt durch Hans Fromm. Tübingen 1969.

Wittgenstein, Ludwig: Philosophische Untersuchungen. Frankfurt/M. ${ }^{3} 1982$.

Wyss, Beat: Habsburgs Panorama. Zur Geschichte des kunsthistorischen Museums in Wien. In: Gert Melville (Hg.): Institutionalität und Symbolisierung. Verstetigungen kultureller Ordnungsmuster in Vergangenheit und Gegenwart. Köln, Weimar, Wien 2001, S. 559-567.

Wyss, Ulrich: Theorie der mittelhochdeutschen L,egendenepik. Erlangen 1973.

Zimmermann, Michel ( $\mathrm{Hg}$.): sauctor et mauctoritas«. Invention et conformisme dans l'écriture médièvale. Paris 2001.

Zingerle, Arnold: Der moralische Körper« der Gesellschaft und sein magischer Schatten. '/ur Pespektivität von Magiebegriffen, am Beispiel von Emile Durkheim. In: Jahrbuch für Volkskunde N.F. 19 (1996), S. 11-25.

\%umthor, Paul: La lettre et la voix. De la littératurer médiévale. Paris 1987. 